\title{
Analysis of exchange terms in a projected extended random phase approximation theory applied to the quasielastic $\left(e, e^{\prime}\right)$ reaction
}

\author{
E. Bauer \\ Departamento de Física, Facultad de Ciencias Exactas, Universidad Nacional de La Plata, La Plata, 1900, Argentina \\ A. Polls and A. Ramos \\ Departament d'Estructura i Constituents de la Matèria, Universitat de Barcelona, Diagonal 647, E-08028, Spain
}

(Received 27 February 1998)

\begin{abstract}
A systematic study of the influence of exchange terms in the longitudinal and transverse nuclear response to quasielastic $\left(e, e^{\prime}\right)$ reactions is presented. The study is performed within the framework of the extended random phase approximation, which in conjuction with a projection method permits a separation of various contributions tied to different physical processes. The calculations are performed in nuclear matter up to second order in the residual interaction for which we take a $(\pi+\rho)$ model with the addition of the LandauMigdal $g^{\prime}$ parameter. Exchange terms are found to be important only for the random-phase-approximationtype contributions around the quasielastic peak. [S0556-2813(98)02508-4]
\end{abstract}

PACS number(s): 21.65.+f, 24.10.Cn, 25.30.Fj

\section{INTRODUCTION}

The nuclear response to an electromagnetic probe is a common tool used to investigate the behavior of the atomic nucleus [1]. In contrast to a hadronic probe it allows a perturbative treatment in the external operator coupling constant. In this work we will concentrate on the study of the nuclear response function for longitudinal and transverse inclusive quasi-elastic electron scattering reactions. These responses are experimentally separated [2-7], showing that a simple model such as the Fermi gas model fails to reproduce the experimental data. The attempts to go beyond this model can be classified in two groups. On one side there are the methods that assume the nucleus as an assembly of noninteracting nucleons with individual properties, such as the charge radius, modified with respect to the vacuum due to the presence of the other nucleons [8-11]. Another option is to explore the possibilities of a rigorous many-body theory [12-36] keeping the nucleons as essential degrees of freedom with the same properties as in the vacuum, before resorting to such exotic effects. This work falls into this second strategy.

Several approaches to the nuclear many-body problem of the nucleus for these processes have been extensively analyzed in the literature [1]. Microscopic many-body theories must deal with short-range correlations (SRC's) originated from the short-range repulsion of the nucleon nucleon $(N N)$ interaction. Variational calculations account for SRC's by introducing a Jastrow correlation factor explicitly in the wave function. In this way, it is possible to define a correlated basis function (CBF) and build a fast converging perturbation theory using this basis. There are recent studies of both longitudinal and transversal responses for nuclear matter in this framework [16]. Alternatively, the effect of SRC's can be incorporated by introducing a well-behaved effective interaction of $G$-matrix type or the standard Landau-Migdal parametrization, with which one can perform perturbation theory to build other correlations, for instance of random- phase approximation (RPA) type. As it is not our aim to describe the full set of approaches, we will comment only on the ones which lead to our particular theory, which is based on the perturbative approach.

A simple way to introduce in the response the nucleonnucleon correlations originated by the residual interaction is by means of the RPA theory, where one-particle-one-hole excitations are summed up to infinite order. Although improving the Fermi gas picture, the RPA approximation is not able to explain some features of the response such as, for instance, the strength in the "dip" region of the transverse response.

An improvement upon the RPA theory consists of allowing the coupling of one-particle-one-hole states to twoparticle-two-hole ones. This corresponds to what has been called final state correlations. Two formalisms study this kind of processes. One is the second RPA (SRPA) theory (see Ref. [15], and references therein) and the other is the Green function scheme of Ref. [17] (see also Refs. [18-21]). The first one introduces final state correlations over the particle-hole bubbles of the RPA theory. In the second one the relationship between forward virtual Compton scattering and inclusive electron scattering is used to construct a onebody approximation to quasielastic electron scattering. In fact, at large momentum transfers, where the effect of longrange correlations is negligible, the SRPA and the opticalmodel Green's function approach should coincide [18].

Both the SRPA and the optical-model Green function approaches use the full residual interaction and allow for manyparticle-many-hole final states. Still in both approaches the one-body external operator is limited to create (or destroy) a one-particle-one-hole pair. Once the external operator is allowed to scatter a particle (or hole), then two-particle-twohole states stemming from ground-state correlations (GSC's) could be activated. The importance of these GSC's are particularly relevant in the dip region for the transverse channel [23]. A theory to calculate the response function which takes into account all the above requirements is already established as the extended RPA (ERPA) theory [25,27]. Still, the appli- 
cation of the ERPA theory is in general a prohibitively large task. In Ref. [30] we developed a projection method which extracts the main ingredients of the ERPA theory. In that work, the scheme was presented and the response was calculated neglecting the exchange part in the matrix elements of the nuclear particle-hole interaction. Therefore it seems necessary to complete the scheme by investigating and establishing the influence of the exchange graphs in the longitudinal and transverse responses of nonrelativistic nuclear matter. Actually, the importance of the exchange terms in RPA theory is a well-known problem which cannot be satisfactorily solved for finite range interactions, although several attempts have been made. In a previous work [31], we developed a simple scheme to evaluate the full antisymmetric RPA series contributing to the nuclear matter response, with the result that exchange contributions are important, specially at low momentum transfer, and cannot be accounted for by simply evaluating the ring (direct) series with an effective $g^{\prime}$ parameter.

In view of the importance of the RPA exchange terms and the ongoing efforts to improve the description of the nuclear response it seems appropriate to explore whether the exchange terms in the remaining types of diagrams contained in the ERPA scheme are also important. In this work, we undertake this task and evaluate for the first time the contribution of the exchange terms to the nuclear response up to second order in the effective interaction.

The paper is organized as follows. In Sec. II we present the formalism. In Sec. III the results for the exchange contributions to the nuclear matter structure function are presented and compared with the corresponding direct ones. Finally, the conclusions are summarized in Sec. IV.

\section{FORMALISM}

The longitudinal $(L)$ and transverse $(T)$ structure functions $S_{L, T}(\mathbf{q}, \hbar \omega)$ are defined as

$$
S_{L, T}(\boldsymbol{q}, \hbar \omega)=-\frac{1}{\pi} \operatorname{Im}\left\langle 0\left|\mathcal{O}_{L, T}^{\dagger} G(\hbar \omega) \mathcal{O}_{L, T}\right| 0\right\rangle,
$$

where $\hbar \omega$ represents the excitation energy and $q$ the magnitude of the three momentum transfer. The nuclear ground state is denoted by $|0\rangle$ while the polarization propagator $G(\hbar \omega)$ is given by

$$
G(\hbar \omega)=\frac{1}{\hbar \omega-H+i \eta}-\frac{1}{\hbar \omega+H-i \eta},
$$

where $H$ is the nuclear Hamiltonian. Explicit forms for the external excitation operators $\mathcal{O}_{L, T}$ are given by

$$
\begin{gathered}
\mathcal{O}_{L}=\sum_{j=1}^{A} \frac{1+\tau_{3}(j)}{2} e^{i \boldsymbol{q} \cdot \boldsymbol{x}_{j}}, \\
\mathcal{O}_{T}=\frac{1}{2 m q} \sum_{j=1}^{A}\left\{\frac{1+\tau_{3}(j)}{2}\left[\boldsymbol{q} \times\left\{\boldsymbol{p}_{j}, e^{i \boldsymbol{q} \cdot \boldsymbol{x}_{j}}\right\}\right]\right. \\
\left.+i \frac{\mu_{s}+\mu_{v} \tau_{3}(j)}{2}\{\boldsymbol{q} \times[\boldsymbol{\sigma}(j) \times \boldsymbol{q}]\} e^{i \boldsymbol{q} \cdot \boldsymbol{x}_{j}}\right\},
\end{gathered}
$$

where $m$ is the nucleonic mass, $\boldsymbol{x}_{j}$ and $\boldsymbol{p}_{j}$ denote the position and momentum operators for individual nucleons, and $\mu_{s}$
$=0.88, \mu_{v}=4.70$ are related to the proton and neutron magnetic moments. In fact, $\mathcal{O}_{L}$ is the charge density operator while $\mathcal{O}_{T}$ is related to the convection and magnetization current density. The structure functions are related to the response functions $R_{L, T}(\boldsymbol{q}, \hbar \omega)$ through the usual dipole electromagnetic form factor $G_{E}(q, \hbar \omega)$

$$
G_{E}(q, \hbar \omega)=\left[1+\frac{(\hbar c q)^{2}-(\hbar \omega)^{2}}{(839 \mathrm{MeV})^{2}}\right]^{-2}
$$

We introduce now the usual projection operator $P$, which projects into $n p n h$ configurations with $n=0,1$, defined with respect to the HF vacuum, which corresponds to the case $n$ $=0$ and is denoted by |\rangle . In addition, two projection operators $Q$ and $R$ are introduced. The action of $Q(R)$ is to project onto the $n p n h$ space with $n$ being an even integer greater or equal to 2 ( $n$ odd greater or equal to 3 ). Explicit expressions are given by

$$
\begin{aligned}
& P=\sum_{n=0,1,}|n\rangle\langle n|, \\
& Q=\sum_{\substack{n \text { even, } \\
n \geqslant 2,}}|n\rangle\langle n|, \\
& R=\sum_{\substack{n \text { odd, } \\
n \geqslant 3,}}|n\rangle\langle n|,
\end{aligned}
$$

where $|n\rangle$ indicates a $n p n h$ configuration.

In the literature only one projection operator, which is the sum of $Q$ and $R$, is usually used. The present separation is done for convenience as it helps to clarify the role of $3 p 3 h$ configurations (see Ref. [30]). It is easy to verify that $P$ $+Q+R=1, \quad P^{2}=P, \quad Q^{2}=Q, \quad R^{2}=R, \quad$ and $P Q=Q P$ $=P R=R P=Q R=R Q=0$.

Inserting the identity in Eq. (1) one obtains

$$
S=S_{P P}+S_{P Q}+S_{Q P}+S_{Q Q}+S_{P R}+S_{R P}+S_{R R}+S_{Q R}+S_{R Q},
$$

where, for simplicity, we have omitted the subscripts $L, T$. The expression for $S_{P P}$ is given by

$$
S_{P P}(\boldsymbol{q}, \hbar \omega)=-\frac{\operatorname{Im}}{\pi}\left\langle 0\left|\mathcal{O}^{\dagger} P G(\hbar \omega) P \mathcal{O}\right| 0\right\rangle,
$$

and similar expressions can be written for $S_{P Q}$, etc. To evaluate the propagators $P G P, P G Q$, etc., one has to solve the following equation:

$$
\mathcal{G} \cdot \mathcal{G}^{-1}=I
$$

where

$$
\mathcal{G}=\left(\begin{array}{lll}
P G P & P G Q & P G R \\
Q G P & Q G Q & Q G R \\
R G P & R G Q & R G R
\end{array}\right), \quad I=\left(\begin{array}{ccc}
P & 0 & 0 \\
0 & Q & 0 \\
0 & 0 & R
\end{array}\right) .
$$

This is an easy task once the properties of the projection operators are employed. Keeping terms up to second order in 
the nuclear interaction, all $3 p 3 h$ final state contributions cancel each other [30]. Thus, from all terms of Eq. (9), only $S_{P P}, S_{Q P}\left(S_{P Q}\right)$, and $S_{Q Q}$ survive:

$$
\begin{aligned}
S_{P P}= & -\frac{\operatorname{Im}}{\pi} \\
& \times\left\langle 0\left|\mathcal{O}^{\dagger} P \frac{1}{\hbar \omega-H-\Sigma^{P Q P}-\operatorname{Re} \Sigma^{P R P}+i \eta} P \mathcal{O}\right| 0\right\rangle \\
S_{Q P}= & -\frac{\operatorname{Im}}{\pi}\langle 0| \mathcal{O}^{\dagger} P \frac{1}{\hbar \omega-H_{0}+i \eta} P H_{\mathrm{res}} Q \\
& \times \frac{1}{\hbar \omega-H_{0}+i \eta} Q \mathcal{O}|0\rangle
\end{aligned}
$$

and

$$
S_{Q Q}=-\frac{\operatorname{Im}}{\pi}\left\langle 0\left|\mathcal{O}^{\dagger} Q \frac{1}{\hbar \omega-H_{0}+i \eta} Q \mathcal{O}\right| 0\right\rangle,
$$

where $S_{Q P}$ equals $S_{P Q}$. The self-energy operators introduced in Eq. (12) are given by

$$
\Sigma^{P Q P}=P H_{\mathrm{res}} Q \frac{1}{\hbar \omega-H_{0}+i \eta} Q H_{\mathrm{res}} P
$$

and

$$
\operatorname{Re} \Sigma^{P R P}=-P H_{\mathrm{res}} R \frac{\mathcal{P}}{\hbar \omega-H_{0}} R H_{\mathrm{res}} P,
$$

where $\mathcal{P}$ denotes the principal value. We have separated the total Hamiltonian $H$ into a one-body part $H_{0}$ and a residual interaction $H_{\text {res }}$.

As pointed out in Ref. [30], there is still a contribution stemming from a $3 p 3 h$ configuration given by the real part of $3 p 3 h$-self-energy insertion $\left(\operatorname{Re} \Sigma^{P R P}\right)$ in Eq. (12). That is, up to second order, no $3 p 3 h$ physical state is possible, but virtual intermediate $3 p 3 h$ configurations produce a shift in the ground-state energy (see Refs. [30] and [26] for more details). The next step is to establish the structure of the ground-state. Including the ground state correlations perturbatively one gets, up to first order in the residual interaction,

$$
|0\rangle=|\rangle-H_{0}^{-1} Q H_{\text {res }} P|\rangle,
$$

with |\rangle being the Hartree Fock ground state. More explicitly, one can write

$$
|0\rangle=|\rangle-\sum_{2} \frac{\left\langle 2\left|H_{\mathrm{res}}\right|\right\rangle}{\varepsilon_{\mathrm{gsc}}}|2\rangle,
$$

where the quantity $\varepsilon_{\text {gsc }}$ refers to the energy of the first-order correction to the ground-state energy.

The aim of this section is to present the formalism showing explicitly antisymmetric matrix elements. The guidelines to obtain analytical expressions are given in Ref. [30], where direct contributions were studied. To complete the scheme, expressions for the exchange self-energy insertions are given in Appendix A and exchange terms to the structure function are presented in Appendix B. Also, in order to simplify the calculation, we will limit ourselves to the case in which the external operator is attached to the same bubble. We will study some exceptions to this as a consequence of antisymmetrization. The three nonvanishing contributions $S_{P P}, S_{Q P}$, and $S_{Q Q}$, will be analyzed separately below. Special attention will be paid to $S_{P P}$ as its structure is very rich and represents the main contribution to the response function.

\section{A. $S_{P P}$ contribution}

Let us carefully analyze all graphs stemming from $S_{P P}$. To do this, we first insert the definition of $P$ given by Eq. (6) into $S_{P P}$ [Eq. (12)]:

$$
\begin{aligned}
S_{P P}= & -\frac{1}{\pi} \operatorname{Im} \sum_{n, n^{\prime}=1 .}\left\langle 0\left|\mathcal{O}^{\dagger}\right| n\right\rangle \\
& \times\left\langle n\left|\frac{1}{\hbar \omega-H_{0}-H_{\mathrm{res}}-\Sigma^{P Q P}-\operatorname{Re} \Sigma^{P R P}+i \eta}\right| n^{\prime}\right\rangle \\
& \times\left\langle n^{\prime}|\mathcal{O}| 0\right\rangle .
\end{aligned}
$$

Using the ground state given by Eq. (18) in the expression and neglecting all third and higher orders terms except the ones with self-energy insertions, one can write

$$
\begin{aligned}
S_{P P}= & S_{P P}{ }^{\text {Lindhard+self-energy }}+S_{P P} \text { first-order RPA } \\
& +S_{P P} \text { second-order RPA }
\end{aligned}
$$

where

$$
\begin{aligned}
S_{P P}^{\text {Lindhard+self-energy }} & =-\frac{1}{\pi} \operatorname{Im} \sum_{1,1^{\prime}}\left\langle\left|\mathcal{O}^{\dagger}\right| 1\right\rangle\left\langle 1\left|\frac{1}{\hbar \omega-H_{0}-\Sigma^{P Q P}-\operatorname{Re} \Sigma^{P R P}+i \eta}\right| 1^{\prime}\right\rangle_{a}\left\langle 1^{\prime}|\mathcal{O}|\right\rangle, \\
S_{P P}^{\text {first-order RPA }} & =-\frac{1}{\pi} \operatorname{Im}\left\{\sum_{1,1^{\prime}}\left\langle\left|\mathcal{O}^{\dagger}\right| 1\right\rangle \frac{1}{\hbar \omega-\varepsilon_{1}+i \eta}\left\langle 1\left|H_{\mathrm{res}}\right| 1^{\prime}\right\rangle_{a} \frac{1}{\hbar \omega-\varepsilon_{1^{\prime}}+i \eta}\left\langle 1^{\prime}|\mathcal{O}|\right\rangle\right. \\
& \left.-2 \sum_{1} \sum_{2} \frac{\left\langle\left|H_{\mathrm{res}}\right| 2\right\rangle_{a}}{\varepsilon_{\mathrm{gsc}}}\left\langle 2\left|\mathcal{O}^{\dagger}\right| 1\right\rangle \frac{1}{\hbar \omega-\varepsilon_{1}+i \eta}\langle 1|\mathcal{O}|\rangle\right\},
\end{aligned}
$$




$$
\begin{aligned}
S_{P P} \text { second-order RPA }= & -\frac{1}{\pi} \operatorname{Im}\left\{\sum_{1,1^{\prime}, 1^{\prime \prime}}\left\langle\left|\mathcal{O}^{\dagger}\right| 1\right\rangle \frac{1}{\hbar \omega-\varepsilon_{1}+i \eta}\left\langle 1\left|H_{\mathrm{res}}\right| 1^{\prime}\right\rangle_{a} \frac{1}{\hbar \omega-\varepsilon_{1^{\prime}}+i \eta}\left\langle 1^{\prime}\left|H_{\mathrm{res}}\right| 1^{\prime \prime}\right\rangle_{a} \frac{1}{\hbar \omega-\varepsilon_{1^{\prime \prime}}+i \eta}\left\langle 1^{\prime \prime}|\mathcal{O}|\right\rangle\right. \\
& -2 \sum_{1,1^{\prime \prime}} \sum_{2} \frac{\left\langle\left|H_{\mathrm{res}}\right| 2\right\rangle_{a}}{\varepsilon_{\mathrm{gsc}}}\left\langle 2\left|\mathcal{O}^{\dagger}\right| 1\right\rangle \frac{1}{\hbar \omega-\varepsilon_{1}+i \eta}\left\langle 1\left|H_{\mathrm{res}}\right| 1^{\prime}\right\rangle_{a} \frac{1}{\hbar \omega-\varepsilon_{1^{\prime}}+i \eta}\left\langle 1^{\prime}|\mathcal{O}|\right\rangle \\
& \left.+\sum_{1} \sum_{2,2^{\prime}} \frac{\left\langle\left|H_{\mathrm{res}}\right| 2\right\rangle_{a}}{\varepsilon_{\mathrm{gsc}}}\left\langle 2\left|\mathcal{O}^{\dagger}\right| 1\right\rangle \frac{1}{\hbar \omega-\varepsilon_{1}+i \eta}\left\langle 1|\mathcal{O}| 2^{\prime}\right\rangle \frac{\left\langle 2\left|H_{\mathrm{res}}\right|\right\rangle_{a}}{\varepsilon_{\mathrm{gsc}}}\right\}
\end{aligned}
$$

and $H_{0}|n\rangle=\varepsilon_{n}|n\rangle$.

From Eqs. (15) and (16) the self-energy insertions read now

$$
\left\langle 1\left|\Sigma^{P Q P}\right| 1^{\prime}\right\rangle=\sum_{2}\left\langle 1\left|H_{\mathrm{res}}\right| 2\right\rangle_{a} \frac{1}{\hbar \omega-\varepsilon_{2}+i \eta}\left\langle 2\left|H_{\mathrm{res}}\right| 1^{\prime}\right\rangle_{a}
$$

and

$$
\left\langle 1\left|\operatorname{Re} \Sigma^{P R P}\right| 1^{\prime}\right\rangle=-\sum_{3}\left\langle 1\left|H_{\mathrm{res}}\right| 3\right\rangle_{a} \frac{\mathcal{P}}{\hbar \omega-\varepsilon_{3}}\left\langle 3\left|H_{\mathrm{res}}\right| 1^{\prime}\right\rangle_{a}
$$

In all expressions we have made explicit indication of antisymmetric matrix elements.

In Fig. 1 some graphs contributing to $S_{P P}$ are shown. Within brackets we have collected direct plus exchange contributions. Let us start by analyzing the contribution to
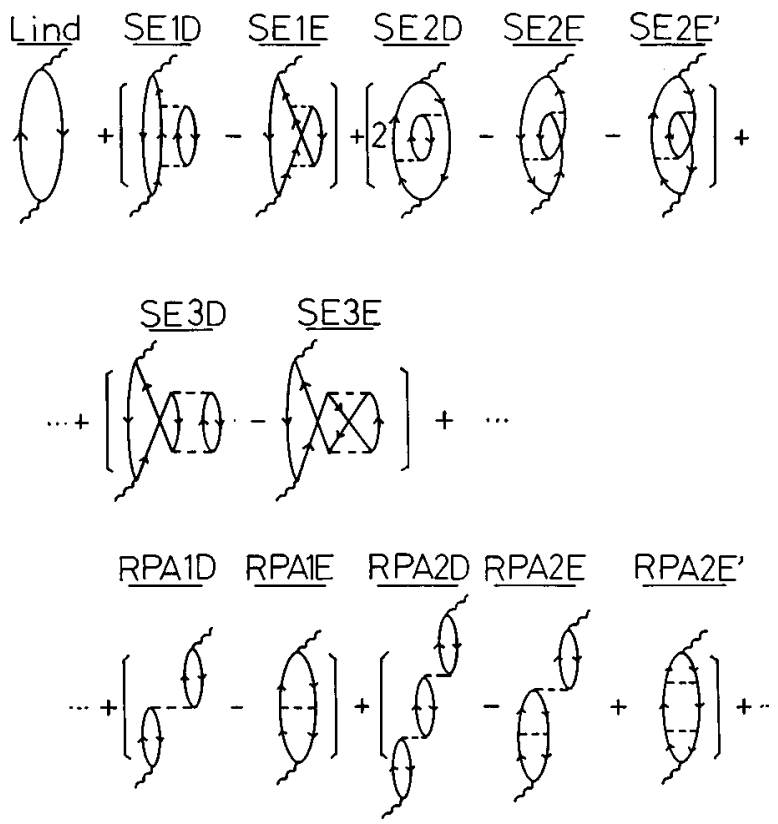

FIG. 1. Goldstone diagrams stemming from Eqs. (21)-(23). In every diagram the wavy lines represent the external probe with energy momentum $(\mathbf{q}, \omega)$. The dashed line is the residual interaction. For simplicity we show only forward-going contributions, where the incoming external probe creates a particle-hole pair. In the backward-going diagrams (not represented here) the probe can also destroy a particle-hole pair.
$S_{P P}$ Lindhard+self-energy. The presence of self-energy operators makes the energy denominators in the right-hand side (RHS) of Eq. (21) nondiagonal in our particle-hole basis. Nondiagonal terms, shown by graphs SE2D, SE2E and SE2E' in Fig. 1 , are evaluated at second order. For diagonal ones, shown by graphs SE1D, SE1E, SE3D, and SE3E in Fig. 1, we first build up an antisymmetric self-energy insertion and then sum it up to infinite order.

The first two orders leading to the RPA response [Eqs. (22) and (23)], are shown by graphs RPA1D to RPA2E' in Fig. 1, where only the forward going contributions are explicitly shown, that is, the ones stemming from the first terms on the right-hand side of the above mentioned equations. If exchange terms were neglected one would be able to sum terms up to infinite order leading to the usual ring series.

As mentioned earlier, in this paper we keep terms up to second order in the evaluation of exchange contributions. However, for RPA-type diagrams we use the method described in Ref. [31], which allows us to effectively sum up the full antisymmetric RPA series. That method is based on splitting the interaction into a pure contact part and a remaining part chosen such that the second-order ring coincides with the full ring series. The pure contact term allows a straightforward evaluation of the antisymmetric RPA series up to infinite order, while only terms up to second order are retained for the remaining part of the interaction.

\section{B. $S_{Q P}$ contribution}

Using the definition of $P$ and $Q$ into $S_{Q P}$ [Eq. (13)] we have

$$
\begin{aligned}
S_{Q P}= & -2 \frac{1}{\pi} \operatorname{Im}\left\{\sum_{1,2,2^{\prime}}\left\langle\left|\mathcal{O}^{\dagger}\right| 1\right\rangle \frac{1}{\hbar \omega-\varepsilon_{1}+i \eta}\left\langle 1\left|H_{\mathrm{res}}\right| 2\right\rangle_{a}\right. \\
& \left.\times \frac{1}{\hbar \omega-\varepsilon_{2}+i \eta}\left\langle 2|\mathcal{O}| 2^{\prime}\right\rangle \frac{\left\langle 2^{\prime}\left|H_{\mathrm{res}}\right|\right\rangle_{a}}{\varepsilon_{\mathrm{gsc}}}\right\} .
\end{aligned}
$$

Note that as $\mathcal{O}$ is a one body operator, it can scatter a particle (or hole) or create (or destroy) a particle-hole pair. That is, the Hartree-Fock ground state is not connected to a $2 p 2 h$ configuration through $\mathcal{O}$.

In Fig. 2 we present the second-order contributions to $S_{Q P}$. As a consequence of antisymmetrization, a direct term where the external operator is attached to a different bubble has come into play (given by graph $S Q P D^{\prime}$ of this figure). Naturally, when we act with the antisymmetrization opera- 

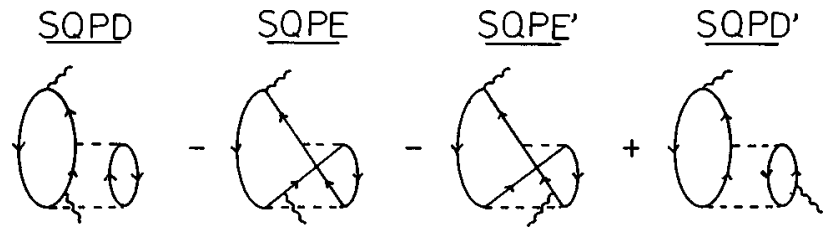

FIG. 2. The same as Fig. 1 but for the $S_{Q P}$ channel given by Eq. (26). The action of the external probe represents the interference between scattering (a particle or a hole) and creating (or destroying) a particle-hole pair.

tor over this term, the same set of graphs appears. We account for this through a factor 2 .

\section{C. $S_{Q Q}$ contribution}

Finally, the expression for $S_{Q Q}$ [Eq. (14)] is simply

$$
\begin{aligned}
S_{Q Q}= & -\frac{1}{\pi} \operatorname{Im}\left\{\sum_{2,2^{\prime}, 2^{\prime \prime}} \frac{\left\langle\left|H_{\mathrm{res}}\right| 2\right\rangle_{a}}{\varepsilon_{\mathrm{gsc}}}\left\langle 2\left|\mathcal{O}^{\dagger}\right| 2^{\prime}\right\rangle \frac{1}{\hbar \omega-\varepsilon_{2^{\prime}}+i \eta}\right. \\
& \left.\times\left\langle 2^{\prime}|\mathcal{O}| 2^{\prime \prime}\right\rangle \frac{\left\langle 2^{\prime \prime}\left|H_{\mathrm{res}}\right|\right\rangle_{a}}{\varepsilon_{\mathrm{gsc}}}\right\} .
\end{aligned}
$$

Here, the only possible action for the external operator is to scatter a particle or a hole.

In Fig. 3, we present the main contributions to $S_{Q Q}$. Graphs $S Q Q 3 D^{\prime}$ presents a direct contribution with the external operator attached to a different bubble, in complete analogy to $S_{Q P}$.

\section{RESULTS FOR NUCLEAR MATTER}

In order to benefit from the advantage of translational invariance, results have been obtained for infinite nuclear matter at normal saturation density corresponding to a Fermi momentum $k_{F}=1.36 \mathrm{fm}^{-1}$. For the residual interaction $H_{\text {res }}$ we assume the $(\pi+\rho)$-exchange model at the static limit with the addition of the Landau Migdal $g^{\prime}$ parameter. In pionic units it reads
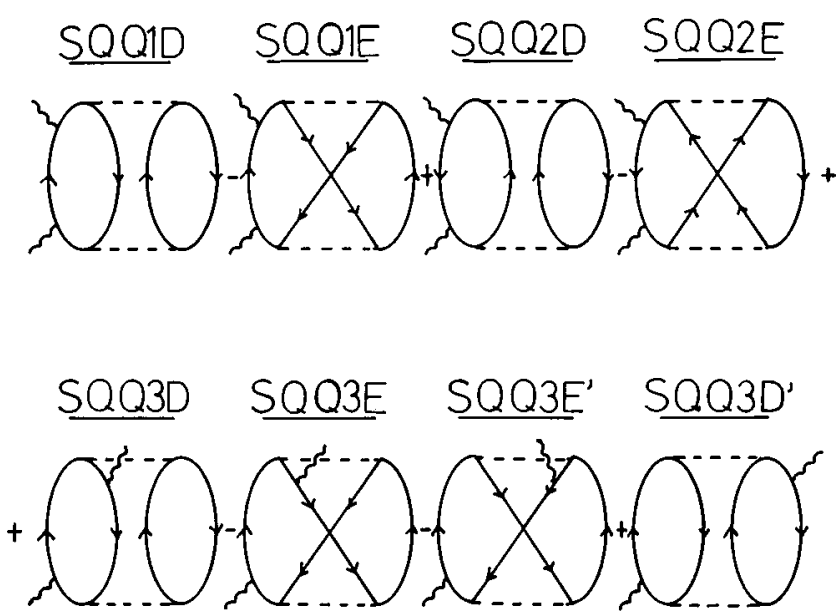

FIG. 3. The same as Fig. 1 but for the $S_{Q Q}$ channel given by Eq. (27). The action of the external probe is to create (or destroy) a particle-hole pair.

$$
H_{\mathrm{res}}(l)=\frac{f_{\pi}^{2}}{\mu_{\pi}^{2}} \Gamma_{\pi}^{2}(l)\left[\tilde{g}^{\prime}(l) \boldsymbol{\tau} \cdot \boldsymbol{\tau}^{\prime} \boldsymbol{\sigma} \cdot \boldsymbol{\sigma}^{\prime}+\tilde{h}^{\prime}(l) \boldsymbol{\tau} \cdot \boldsymbol{\tau}^{\prime} \boldsymbol{\sigma} \cdot \hat{l} \boldsymbol{\sigma}^{\prime} \cdot \hat{l}\right],
$$

with

$$
\begin{gathered}
\tilde{g}^{\prime}(l)=g^{\prime}-\frac{\Gamma_{\rho}^{2}(l)}{\Gamma_{\pi}^{2}(l)} C_{\rho} \frac{l^{2}}{l^{2}+\mu_{\rho}^{2}}, \\
\tilde{h}^{\prime}(l)=-\frac{l^{2}}{l^{2}+\mu_{\pi}^{2}}+\frac{\Gamma_{\rho}^{2}(l)}{\Gamma_{\pi}^{2}(l)} C_{\rho} \frac{l^{2}}{l^{2}+\mu_{\rho}^{2}},
\end{gathered}
$$

where $\mu_{\pi} \hbar c\left(\mu_{\rho} \hbar c\right)$ is the pion (rho) rest mass and $C_{\rho}$ $=2.18$. For the form factor of the $\pi N N(\rho N N)$ vertex we have taken

$$
\Gamma_{\pi, \rho}(l)=\frac{\Lambda_{\pi, \rho}^{2}-\left(\mu_{\pi, \rho} \hbar c\right)^{2}}{\Lambda_{\pi, \rho}^{2}+(\hbar c l)^{2}},
$$

with $\Lambda_{\pi}=1.3 \mathrm{GeV}$ and $\Lambda_{\rho}=2 \mathrm{GeV}$. The role of the $g^{\prime}$ parameter is to account for short-range correlations. Note that for a pure contact interaction exchange contributions have been traditionally included in the RPA series by a redefinition of the Landau-Migdal parameters. In particular, standard $g^{\prime}$ values range from 0.7 to 0.95 but, when redefined to account for antisymmetric terms, the values are lowered and range from 0.5 to 0.7 [37]. As we evaluate explicitly exchange graphs a standard $g^{\prime}$ value from 0.7 to 0.95 should be used. We have employed the value $g^{\prime}=0.7$. In addition, in all the diagrams considered in our calculations, the nucleon lines have been dressed in an average way by taking a momentum-independent effective mass value of $\mathrm{m}^{*} / \mathrm{m}$ $=0.85$.

From Eqs. (21)-(27), explicit expressions for the structure functions in nuclear matter can be obtained, where the sums over the different configurations are replaced by multidimensional integrals. The expressions of the direct terms were reported in Ref. [30] and will not be repeated here. In Appendix A we give the exchange self-energy insertions appearing in Eq. (21) [see Eqs. (24) and (25)], while in Appendix B the exchange contributions to Eqs. (21)-(23) and (26) -(27) are shown (see also Figs. 1-3). The multiple integrations have been performed using a Monte Carlo technique.

Let us analyze the three nonvanishing contributions $S_{P P}, S_{Q P}$, and $S_{Q Q}$ to the response. We follow the notation already shown in Figs. 1-3.

Tables I and II give the results for the $S_{P P}$ channel. In Table I we compare all direct and exchange contributions from self-energy insertions. To avoid divergencies, diagonal self-energy insertions are evaluated up to infinite order [24]. To do this, an average over the hole momentum of the bubble where the self-energy is attached should be done. This procedure is outlined in Appendix A. From the table it is clear that, although small in general, the exchange diagrams can amount to a non-negligible fraction of the direct ones, especially at energies around and below the quasiparticle peak. This is also visualized in Fig. 4, where the structure function including only the direct self-energy diagrams 
TABLE I. Free and self-energy contributions to the longitudinal and transverse structure function. All results are for nuclear matter at momentum transfer $q=410 \mathrm{MeV} / c$ in units of $10^{-5} \mathrm{MeV}^{-1} \mathrm{fm}^{-3}$. The first column represents the energy transfer in $\mathrm{MeV}$. Column Lind. represents the free structure function. Columns SE13 give the direct (D) and exchange (E) contribution to the diagonal part of the self-energy up to infinite order. Their first contributions are the graphs SE1D, SE3D, SE1E, and SE3E of Fig. 1. Columns SE2D, SE2E, and SE2E' are the nondiagonal selfenergy contributions to the structure function as shown in Fig. 1. The last column is the sum of all these contributions given by Eq. (21).

\begin{tabular}{lccccccc}
\hline \hline$\hbar \omega$ & \multicolumn{7}{c}{ Longitudinal } \\
$(\mathrm{MeV})$ & Lind. & SE13D & SE13E & SE2D & SE2E & SE2E' & $S^{\text {Lind+SE }}$ \\
\hline 50.0 & 38.729 & -1.269 & 0.715 & -1.570 & 0.185 & 0.071 & 36.862 \\
100.0 & 46.106 & -4.390 & 0.599 & -0.559 & 0.374 & 0.159 & 42.289 \\
150.0 & 41.357 & -5.949 & 0.397 & 1.085 & -0.096 & -0.050 & 36.743 \\
200.0 & 24.481 & -3.250 & 0.111 & 2.219 & -0.045 & -0.027 & 23.490 \\
250.0 & 0.000 & 2.971 & -0.127 & 0.521 & -0.004 & -0.005 & 3.357 \\
& & \multicolumn{7}{c}{ Transverse } & & & \\
\hline 50.0 & 60.380 & -1.938 & 1.112 & -3.205 & 0.037 & 0.181 & 56.566 \\
100.0 & 72.299 & -6.943 & 0.946 & -0.604 & 0.119 & 0.004 & 65.821 \\
150.0 & 64.610 & -9.354 & 0.621 & 2.575 & -0.071 & -0.232 & 58.149 \\
200.0 & 37.739 & -4.948 & 0.164 & 5.337 & -0.082 & -0.357 & 37.853 \\
250.0 & 0.000 & 4.587 & -0.195 & 0.726 & -0.006 & -0.079 & 5.032 \\
\hline \hline
\end{tabular}

(long-dashed line) is compared to that containing, in addition, the exchange ones (full line) for both the longitudinal (upper part) and the transverse (lower part) channels. The short-dashed line is the free structure function calculated with an effective mass of value of $m^{*} / m=0.85$. As has been observed before [16,32-34], the dressing of the nucleon lines by self-energy insertions smears out the structure function, moving strength out from the quasiparticle peak to the high missing energy region.

Notice that in the diagrams shown in Figs. 1-3 we have not explicitly included the first order self-energy insertion on the fermion propagators. Instead of this, we preferred to use a Lindhard function calculated with an effective mass $m^{*}$ in the nucleon propagators and, therefore, already containing in an average way the effects of those self-energy insertions

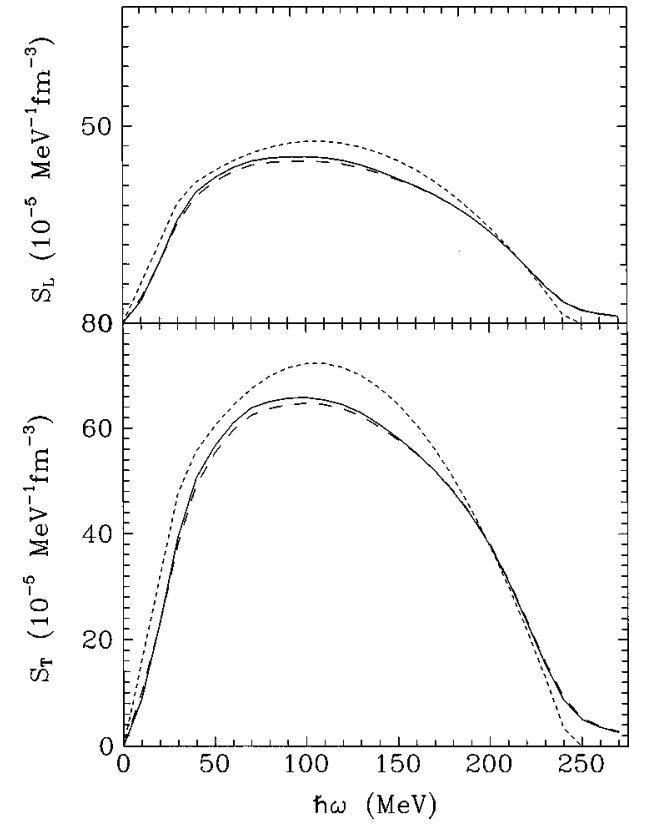

FIG. 4. Self-energy contributions to the longitudinal (upper part) and transverse (lower part) structure function of nuclear matter at momentum transfer $q=410 \mathrm{MeV} / c$. Short-dashed line: Lindhard function using an effective mass $m * / m=0.85$. Long-dashed line: effect of adding the direct self-energy terms. Full line: effect of adding the direct and exchange self-energy terms.

propagated to all orders. Actually, the use of $m^{*}$ is equivalent to having a real and energy-independent self-energy parametrized by a function quadratic in the momentum of the nucleon. These mean field single-particle states define the HF ground state and the basis in which the perturbation theory has been constructed. The second-order self-energy diagrams SE1D,SE3D,SE1E,SE3E are responsible for the appearance of an imaginary part which yields a width to the nucleon lines, which in turn is responsible for the observed spreading in the nuclear response.

In Table II we analyze the RPA-type correlations showing explicit results for first- and second-order contributions. This is done for the transverse channel as RPA-type correlations are zero for the longitudinal one due to the election of our interaction with no $f$ or $f^{\prime}$ Landau-Migdal terms. In the last column we also present the results for RPA correlations

TABLE II. RPA-type contributions to the transverse structure function in units of $10^{-5} \mathrm{MeV}^{-1} \mathrm{fm}^{-3}$ for nuclear matter at momentum transfer $q=410 \mathrm{MeV} / c$. Columns RPA1D (RPA1E) and RPA2D $\left[R P A 2\left(E+E^{\prime}\right)\right]$ are the first- and second-order direct (exchange) part to the RPA response, respectively. The notation is the same as in Fig. 1. Note that in that figure only forward-going contributions are shown while the present results contain both forward and backward-going contributions. Column RPA12D+E is the sum of all first- and second-order contributions [given by Eqs. (22) and (23)]. Finally, column $\mathrm{RPA}_{\text {ant }}$ is the result for a full antisymmetric RPA using the formalism given in Ref. [31].

\begin{tabular}{lcccccc}
\hline \hline $\begin{array}{l}\hbar \omega \\
(\mathrm{MeV})\end{array}$ & RPA1D & RPA1E & RPA2D & RPA2 $\left(\mathrm{E}+\mathrm{E}^{\prime}\right)$ & RPA12D+E & RPA $_{\text {ant }}$ \\
\hline 50.0 & -30.233 & 8.844 & 9.028 & -2.912 & -15.273 & -19.527 \\
100.0 & -13.801 & 4.209 & -3.098 & 0.914 & -11.775 & -9.988 \\
150.0 & 5.122 & -1.132 & -3.441 & 1.204 & 1.753 & 3.307 \\
200.0 & 10.354 & -3.206 & 1.468 & -0.466 & 8.150 & 7.186 \\
250.0 & 0.000 & 0.000 & 0.000 & 0.000 & 0.000 & 0.000 \\
\hline \hline
\end{tabular}




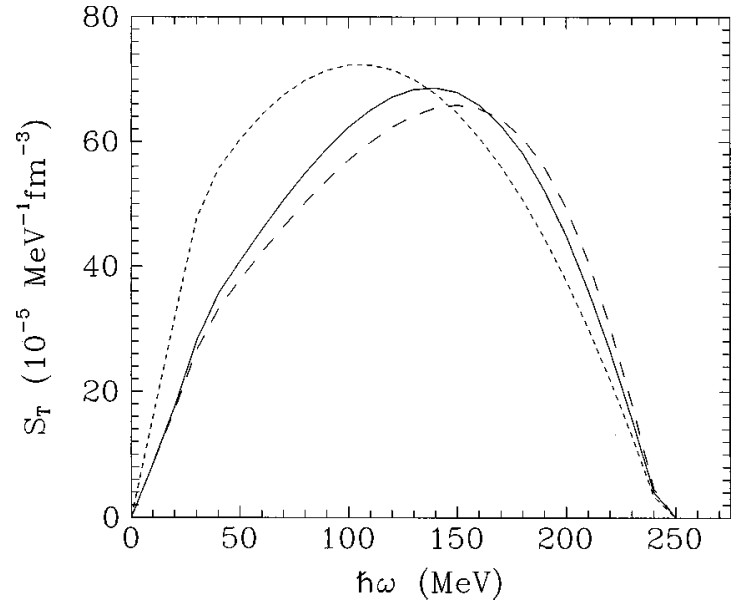

FIG. 5. RPA contributions to the transverse structure function of nuclear matter at $q=410 \mathrm{MeV} / c$. Short-dashed line: Lindhard function $\left(m^{*} / m=0.85\right)$. Long-dashed line: direct ring diagrams. Full line: full RPA structure function including the exchange terms to all orders.

when exchange contributions are included up to infinite order, following the scheme of Ref. [31]. From Table II is clear that exchange terms of RPA type are very important. Their size is comparable (even bigger) to other direct diagrammatic contributions to the structure function and, therefore, they should not be neglected. Given the magnitude of these RPA exchange terms, the differences between the next-to-last and last columns of Table II also suggest that it is important to sum them up to infinite order as was done in Ref. [31]. The effect of the RPA diagrams in the transverse structure function is also displayed in Fig. 5, where the full antisymmetric RPA series (full line) is compared to the direct ring series (long-dashed line). We observe that the transfer of strength from the low- to the high-energy region typical of the polarization (ring) diagrams is partly restored by the incorporation of the exchange diagrams.

In Table III, we study the $S_{Q P}$ channel. In this kind of graph the external operator creates (or destroys) a particle-
TABLE III. Longitudinal and transverse $S_{Q P}$-type structure function in units of $10^{-5} \mathrm{MeV}^{-1} \mathrm{fm}^{-3}$ for nuclear matter at momentum transfer $q=410 \mathrm{MeV} / c$. The notation $S Q P D$ to $S Q P D^{\prime}$ is the same as in Fig. 2. Column $\mathrm{SQP}_{\mathrm{ant}}$ is the sum of all contributions.

\begin{tabular}{lccccc}
\hline \hline \multicolumn{5}{c}{ Longitudinal } \\
$\hbar \omega$ & $S Q P D$ & $S Q P E$ & $S Q P E^{\prime}$ & $S Q P D^{\prime}$ & $S Q P_{\text {ant }}$ \\
$(\mathrm{MeV})$ & & & & & \\
\hline 50.0 & 4.950 & -0.003 & -0.077 & 1.101 & 5.971 \\
100.0 & 4.136 & -0.016 & -0.110 & 1.142 & 5.152 \\
150.0 & 3.116 & -0.055 & -0.075 & 0.589 & 3.575 \\
200.0 & 1.598 & -0.037 & -0.007 & 0.234 & 1.789 \\
250.0 & -1.284 & 0.004 & 0.022 & -0.058 & -1.316 \\
& \multicolumn{5}{c}{ Transverse } \\
\hline 50.0 & 7.469 & -0.604 & -0.101 & 1.324 & 8.089 \\
100.0 & 5.412 & -0.672 & -0.144 & 1.374 & 5.969 \\
150.0 & 3.656 & -0.436 & -0.098 & 0.928 & 4.049 \\
200.0 & 0.906 & -0.001 & -0.009 & 0.491 & 1.387 \\
250.0 & -3.652 & 0.341 & 0.029 & -0.073 & -3.356 \\
\hline \hline
\end{tabular}

hole pair and scatters a particle (or hole). We have evaluated the case where the external operator scatters a particle. The case where it scatters a hole is negligible as can be found in Ref. [30]. As mentioned above, due to the action of the antisymmetrization operator a graph where the external operator is attached to different bubbles has to be considered. This graph is $S Q P D^{\prime}$ in Fig. 2 and has some influence as can be seen from Table III. The other exchange graphs are negligible.

In Table IV the results for the $S_{Q Q}$ channel are shown. As for the $S_{Q P}$ channel, exchange graphs are very small. The importance of the ground-state correlation diagrams is clearly seen in Fig. 6, where the full lines represent the addition of $S_{Q P}+S_{Q Q}$ to the long-dashed lines, which contain the $S_{P P}$ contributions. In the longitudinal channel (upper part), only the self-energy terms contribute to $S_{P P}$, while the

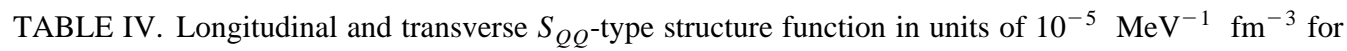
nuclear matter at momentum transfer $q=410 \mathrm{MeV} / c$. The notation $S Q Q 1 D$ to $S Q Q 3 D$ is the same as in Fig. 3. $S Q Q 3 E_{\text {tot }}$ is the sum of $S Q Q 3 E, S Q Q 3 E^{\prime}$, and $S Q Q 3 D^{\prime}$ from the same figure. Column $S Q Q_{\text {ant }}$ is the sum of all contributions.

\begin{tabular}{lccccccc}
\hline \hline \multicolumn{7}{c}{ ¿ } & \multicolumn{7}{c}{ Longitudinal } \\
$(\mathrm{MeV})$ & $S Q Q 1 D$ & $S Q Q 1 E$ & $S Q Q 2 D$ & $S Q Q 2 E$ & $S Q Q 3 D$ & $S Q Q 3 E_{\text {tot }}$ & $S Q Q_{\text {ant }}$ \\
\hline 50.0 & & & & & & & \\
100.0 & 4.692 & -0.042 & 2.455 & -0.074 & -0.552 & 0.041 & 6.520 \\
150.0 & 8.535 & -0.327 & 1.428 & -0.046 & -0.629 & 0.042 & 9.003 \\
200.0 & 9.807 & -0.344 & 0.661 & -0.019 & -0.299 & 0.029 & 9.834 \\
250.0 & 10.426 & -0.278 & 0.310 & -0.008 & -0.220 & 0.020 & 10.249 \\
& 10.342 & -0.167 & 0.047 & -0.003 & -0.113 & 0.011 & 10.118 \\
50.0 & & & & & & & \\
100.0 & 12.673 & -0.307 & 2.493 & -0.027 & -2.230 & 0.017 & 12.617 \\
150.0 & 15.153 & -0.313 & 1.179 & -0.007 & -1.848 & 0.014 & 14.178 \\
200.0 & 17.352 & -0.263 & 0.559 & -0.003 & -0.467 & 0.012 & 17.188 \\
250.0 & 16.551 & -0.167 & 0.090 & -0.001 & -0.271 & 0.006 & 16.208 \\
\hline \hline
\end{tabular}




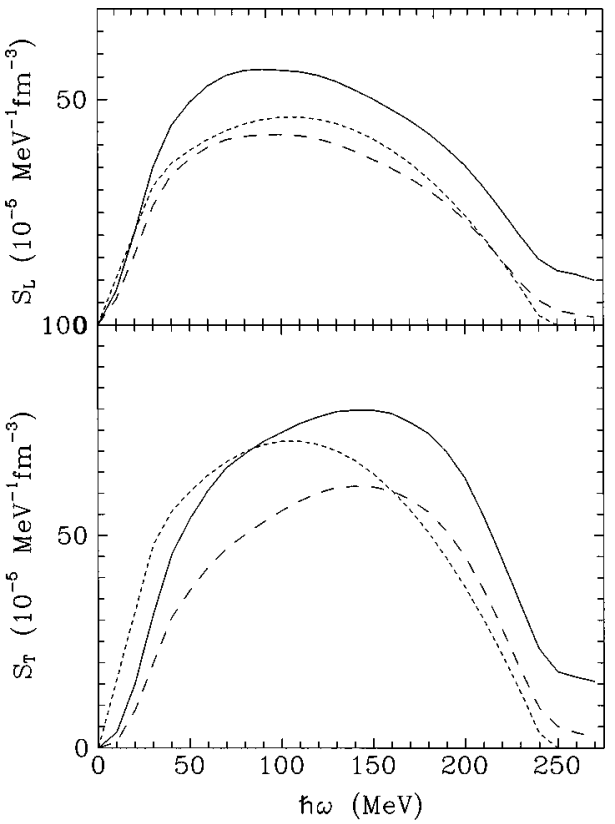

FIG. 6. Contribution of ground-state correlations to the longitudinal (upper part) and transverse (lower part) structure function of nuclear matter at momentum transfer $q=410 \mathrm{MeV} / c$. Shortdashed line: Lindhard function $(m * / m=0.85)$. Long-dashed line: $S_{P P}$ structure function. Full line: inclusion of the ground-state correlation diagrams to the $S_{P P}$ structure function.

transverse channel (lower part) contains, in addition, the RPA-type correlations. In view of these results it is clear that incorporating the $S_{Q P}$ and $S_{Q Q}$ channels is necessary in any perturbative calculation of the nuclear matter response as observed in Ref. [30]. Having established in this work the smallness of the corresponding exchange terms is particulary interesting, since the calculation of these channels can be restricted to the direct graphs thus avoiding a great deal of numerical computation.
In addition our findings also support the idea that the use of an effective $g^{\prime}$ to account for exchange terms in the nuclear response is not appropriate. This point was already raised in Ref. [31], where we built a prescription to calculate the full antisymmetric RPA series of the nuclear response. In that work, we showed that the use of a standard average prescription for $g^{\prime}$ was not able to reproduce the RPA antisymmetric response, especially for intermediate values of the momentum transfer. Using the average $g^{\prime}$ for calculating the other types of correlations would not be appropriate either because we have shown that they are basically dominated by the direct contributions. This is visualized in Table $\mathrm{V}$ where, changing the $g^{\prime}$ parameter to an effective value of $g^{\prime}=0.5$ does not, in the first place, reproduce the antisymmetric RPA response contained implicitly in the first column of the transverse part (a point already raised in Ref. [31]) but also induces non-negligible modifications in the other contributions, especially those related to ground-state correlations (compare the next-to-last and last columns).

From our study we conclude that the nuclear response is basically dominated by the direct diagrams and the exchange ones only need to be considered (and to all orders) for the RPA-type correlations.

\section{CONCLUSIONS}

A projection method to extract the main contributions of the ERPA theory with the explicit inclusion of exchange terms has been developed. This work is a continuation of a previous one [30] in which only direct terms were studied. Here we have tried to clarify the importance of exchange terms of the particle-hole interaction, by performing a quantitative analysis of their influence in the nuclear response.

The projection method classifies the contributions to the nuclear structure function into three channels, called $S_{P P}, S_{Q P}$, and $S_{Q Q}$ with $P$ and $Q$ being projections opera-

TABLE V. Comparison between our results and the corresponding direct values calculated with a modified value of the $g^{\prime}$ Landau-Migdal parameter $\left(g^{\prime}=0.5\right)$ to partially reproduce the exchange contributions. Our results are given by $S P P_{\text {ant }}, S Q P_{\text {ant }}$, and $S Q Q_{\text {ant }}$ where a value $g^{\prime}=0.7$ was employed with the explicit inclusion of exchange graphs. The results, given in units of $10^{-5} \mathrm{MeV}^{-1} \mathrm{fm}^{-3}$, are for nuclear matter at momentum transfer $q=410 \mathrm{MeV} / c$.

\begin{tabular}{|c|c|c|c|c|c|c|}
\hline & & & Longitud & & & \\
\hline $\begin{array}{l}\hbar \omega \\
(\mathrm{MeV})\end{array}$ & $S P P_{\text {ant }}$ & $S P P_{\mathrm{dir}}, g^{\prime}=0.5$ & $S Q P_{\text {ant }}$ & $S Q P_{\mathrm{dir}, g^{\prime}=0.5}$ & $S Q Q_{\text {ant }}$ & $S Q Q_{\mathrm{dir}, g^{\prime}=0.5}$ \\
\hline 50.0 & 36.862 & 36.409 & 5.971 & 5.034 & 6.520 & 2.529 \\
\hline 100.0 & 42.289 & 43.256 & 5.152 & 5.442 & 9.003 & 4.995 \\
\hline 150.0 & 36.743 & 36.430 & 3.575 & 4.312 & 9.834 & 5.971 \\
\hline 200.0 & 23.490 & 19.059 & 1.789 & 0.966 & 10.249 & 6.291 \\
\hline 250.0 & 3.357 & 2.393 & -1.316 & -1.336 & 10.118 & 6.015 \\
\hline $\begin{array}{l}\hbar \omega \\
(\mathrm{MeV})\end{array}$ & $S P P_{\text {ant }}$ & $S P P_{\mathrm{dir}, g^{\prime}}=0.5$ & $\begin{array}{l}\text { Transve } \\
S Q P_{\text {ant }}\end{array}$ & $S Q P_{\mathrm{dir}, g^{\prime}=0.5}$ & $S Q Q_{\mathrm{ant}}$ & $S Q Q_{\mathrm{dir}, g^{\prime}=0.5}$ \\
\hline 50.0 & 37.039 & 47.428 & 7.109 & 5.533 & 8.762 & 5.049 \\
\hline 100.0 & 55.833 & 63.337 & 5.362 & 5.719 & 12.617 & 9.092 \\
\hline 150.0 & 61.456 & 59.066 & 4.049 & 3.864 & 14.178 & 10.814 \\
\hline 200.0 & 45.039 & 33.882 & 1.727 & 1.392 & 17.188 & 11.586 \\
\hline 250.0 & 5.032 & 3.719 & -3.356 & -1.883 & 16.208 & 10.800 \\
\hline
\end{tabular}


tors defined in Sec. I. This separation permits the study of the effects of the different types of correlations. In this sense, $S_{P P}$ represents final state correlations, $S_{Q Q}$ ground-state correlations, and $S_{Q P}$ the interference between them. Through the analysis of our results we can conclude that all types of correlations are important and should be considered when one studies the nuclear response.

After this statement, the problem is the big number of graphs which should be evaluated when the exchange part of the nucleon-nucleon interaction is retained. Before the numerical calculation we can see no reason to neglect any contribution. Our calculations show that for final state correlations, i.e., self-energy insertions and mainly RPA-type correlations, the exchange graphs are relevant in agreement with Refs. [37,38]. On the other hand, they can be neglected for ground-state correlations. Also, within the energymomentum region under consideration, exchange terms can not be parametrized by a redefinition of the $g^{\prime}$ parameter.
It is also important to stress that the interaction employed and in particular the value for $g^{\prime}$, comes from parametrizations of processes at a lower-energy-momentum region than the ones considered here. Those values do not necessarily hold for us. Also from Table V, we see that a small change in one parameter can produce a noticeable change in the structure function. In any case, our objective was not to search for the optimal paremeters that produce good agreement with the experimental data but to careful analyze the exchange diagrams and our conclusions should remain valid in a wide variety of situations.

In summary, our study shows that the nuclear response is basically dominated by the direct diagrams, the most relevant being those of Figs. 1-3, and the exchange contributions only need to be considered (and to all orders) for the RPAtype correlations, which can be evaluated using the prescription of Ref. [31].

\section{APPENDIX A}

In this appendix we show explicit expressions for exchange contributions to the self-energy insertions Direct contributions can be found in Ref. [30]. Exchange self-energy insertions from Eqs. (24) and (25) (which contribute to graphs SE1E and SE3E of Fig. 1, respectively) are given by

$$
\begin{aligned}
{\left[\Sigma^{P Q P}(\boldsymbol{Q}, \nu, \boldsymbol{h})\right]^{\mathrm{part} \text { exch }}=} & -\frac{1}{(2 \pi)^{4}}\left(\frac{f_{\pi}^{2}}{4 \pi \hbar c}\right)^{2} \frac{m c^{2} k_{F}^{4}}{\mu_{\pi}^{4}} \int d^{3} k \int d^{3} k^{\prime} \theta(|\boldsymbol{h}+\boldsymbol{Q}-\boldsymbol{k}|-1) \theta\left(1-\left|\boldsymbol{h}+\boldsymbol{Q}-\boldsymbol{k}-\boldsymbol{k}^{\prime}\right|\right) \theta\left(\left|\boldsymbol{h}+\boldsymbol{Q}-\boldsymbol{k}^{\prime}\right|-1\right) \\
& \times \Gamma_{\pi}^{2}(k) \Gamma_{\pi}^{2}\left(k^{\prime}\right)\left\{3 \tilde{g}^{\prime 2}-\left[2\left(\hat{\boldsymbol{k}} \cdot \widehat{\boldsymbol{k}^{\prime}}\right)^{2}-1\right] \tilde{h}^{\prime 2}+2 \tilde{g}^{\prime} \tilde{h}^{\prime}\right\} \frac{1}{\nu-\left(Q^{2} / 2+\boldsymbol{h} \cdot \boldsymbol{Q}-\boldsymbol{k} \cdot \boldsymbol{k}^{\prime}\right)+i \eta}
\end{aligned}
$$

and

$$
\begin{aligned}
{\left[\operatorname{Re} \Sigma^{P R P}(\boldsymbol{Q}, \nu, \boldsymbol{h})\right]^{\mathrm{part} \text { exch }}=} & \frac{1}{(2 \pi)^{4}}\left(\frac{f_{\pi}^{2}}{4 \pi \hbar c}\right)^{2} \frac{m c^{2} k_{F}^{4}}{\mu_{\pi}^{4}} \int d^{3} k \int d^{3} k^{\prime} \theta(1-|\boldsymbol{h}+\boldsymbol{Q}-\boldsymbol{k}|) \theta\left(\left|\boldsymbol{h}+\boldsymbol{Q}-\boldsymbol{k}-\boldsymbol{k}^{\prime}\right|-1\right) \theta\left(1-\left|\boldsymbol{h}+\boldsymbol{Q}-\boldsymbol{k}^{\prime}\right|\right) \\
& \times \Gamma_{\pi}^{2}(k) \Gamma_{\pi}^{2}\left(k^{\prime}\right)\left\{3 \tilde{g}^{\prime 2}-\left[2\left(\hat{\boldsymbol{k}} \cdot \widehat{\boldsymbol{k}^{\prime}}\right)^{2}-1\right] \tilde{h}^{\prime 2}+2 \tilde{g}^{\prime} \widetilde{h}^{\prime}\right\} \frac{1}{\nu-\left(Q^{2} / 2+\boldsymbol{h} \cdot \boldsymbol{Q}+\boldsymbol{k} \cdot \boldsymbol{k}^{\prime}\right)}
\end{aligned}
$$

We have used dimensionless quantities $Q=q / k_{F}$ and $\nu=\hbar \omega / 2 \varepsilon_{F} ; k_{F}$ and $\varepsilon_{F}$ being the Fermi momentum and energy, respectively.

In order to simplify the calculation, it is a good approximation to eliminate the dependence on the hole momentum, using an average procedure (see Ref. [30]), as follows:

$$
\Sigma^{P Q(R) P}(\boldsymbol{Q}, \nu) \equiv \frac{1}{\frac{4}{3} \pi} \int d^{3} h \Sigma^{P Q(R) P}(\boldsymbol{Q}, \nu, \boldsymbol{h})
$$

\section{APPENDIX B}

In this appendix we show explicit expressions for exchange contributions to the structure function. Direct contributions can be found in Ref. [30].

Let us first consider the $S_{P P}$ channel. Graph SE2E of Fig. 1 [see Eq. (21)] is given by 


$$
\begin{aligned}
{\left[S_{S E 1}(\boldsymbol{Q}, \nu)\right]_{L, T}=} & -\frac{A}{(2 \pi)^{5}}\left(\frac{f_{\pi}^{2}}{4 \pi \hbar c}\right)^{2} \frac{3\left(m c^{2}\right)^{3}}{2\left(\hbar c \mu_{\pi}\right)^{4}} \int d^{3} h \int d^{3} k \int d^{3} k^{\prime} \mathcal{O} \mathcal{V}_{(L, T), \mathrm{SE} 2 \mathrm{E}} \theta(1-|\boldsymbol{h}|) \theta(|\boldsymbol{h}+\boldsymbol{Q}|-1) \theta\left(1-\left|\boldsymbol{h}+\boldsymbol{k}^{\prime}\right|\right) \\
& \times \theta\left(\left|\boldsymbol{h}+\boldsymbol{k}+\boldsymbol{k}^{\prime}\right|-1\right) \theta(1-|\boldsymbol{h}+\boldsymbol{k}|) \theta(|\boldsymbol{h}+\boldsymbol{k}+\boldsymbol{Q}|-1)\left(\frac{-1}{\pi} \operatorname{Im}\right) \\
& \times\left[\frac{1}{\nu-\left(Q^{2} / 2+\boldsymbol{Q} \cdot \boldsymbol{h}\right)+i \eta} \frac{1}{\nu-\left(Q^{2} / 2+\boldsymbol{Q} \cdot \boldsymbol{h}+\boldsymbol{k} \cdot \boldsymbol{k}^{\prime}\right)+i \eta} \frac{1}{\nu-\left[Q^{2} / 2+\boldsymbol{Q} \cdot(\boldsymbol{h}+\boldsymbol{k})\right]+i \eta}\right]
\end{aligned}
$$

Graph SE2E' of Fig. 1 [see Eq. (21)]:

$$
\begin{aligned}
{\left[S_{{\mathrm{SE} 2 \mathrm{E}^{\prime}}^{\prime}}(\boldsymbol{Q}, \nu)\right]_{L, T}=} & -\frac{A}{(2 \pi)^{5}}\left(\frac{f_{\pi}^{2}}{4 \pi \hbar c}\right)^{2} \frac{3\left(m c^{2}\right)^{3}}{2\left(\hbar c \mu_{\pi}\right)^{4}} \int d^{3} h \int d^{3} k \int d^{3} k^{\prime} \mathcal{O} \mathcal{V}_{(L, T), \mathrm{SE} 2 \mathrm{E}^{\prime}} \theta(1-|\boldsymbol{h}|) \theta(|\boldsymbol{h}+\boldsymbol{Q}|-1) \theta(1-|\boldsymbol{h}+\boldsymbol{k}|) \\
& \times \theta\left(1-\left|\boldsymbol{h}+\boldsymbol{k}+\boldsymbol{k}^{\prime}+\boldsymbol{Q}\right|\right) \theta\left(\left|\boldsymbol{h}+\boldsymbol{k}^{\prime}+\boldsymbol{Q}\right|\right) \theta(|\boldsymbol{h}+\boldsymbol{k}+\boldsymbol{Q}|-1)\left(\frac{-1}{\pi} \mathrm{Im}\right) \\
& \times\left[\frac{1}{\nu-\left(Q^{2} / 2+\boldsymbol{Q} \cdot \boldsymbol{h}\right)+i \eta} \frac{1}{\nu-\left(Q^{2} / 2+\boldsymbol{Q} \cdot \boldsymbol{h}-\boldsymbol{k} \cdot \boldsymbol{k}^{\prime}\right)+i \eta} \frac{1}{\nu-\left[Q^{2} / 2+\boldsymbol{Q} \cdot(\boldsymbol{h}+\boldsymbol{k})\right]+i \eta}\right]
\end{aligned}
$$

where

$$
\mathcal{O} \mathcal{V}_{L, \mathrm{SE} 2 \mathrm{E}}=\mathcal{O} \mathcal{V}_{L, \mathrm{SE} 2 \mathrm{E}^{\prime}}=\Gamma_{\pi}^{2}(k) \Gamma_{\pi}^{2}\left(k^{\prime}\right)\left\{3 \tilde{g}^{\prime 2}-\left[2\left(\hat{\boldsymbol{k}} \cdot \widehat{\boldsymbol{k}^{\prime}}\right)^{2}-1\right] \tilde{h}^{\prime 2}+2 \tilde{g}^{\prime} \tilde{h}^{\prime}\right\}
$$

and

$$
\begin{aligned}
\mathcal{O} \mathcal{V}_{T, \mathrm{SE} 2 \mathrm{E}}= & \mathcal{O} \mathcal{V}_{T, \mathrm{SE}_{2} \mathrm{E}^{\prime}} \\
= & \left(\frac{\hbar c k_{F}}{2 m c^{2}}\right)^{2} \Gamma_{\pi}^{2}(k) \Gamma_{\pi}^{2}\left(k^{\prime}\right)\left\{4\{\boldsymbol{h} \cdot(\boldsymbol{h}+\boldsymbol{k})-(\hat{\boldsymbol{Q}} \cdot \boldsymbol{h})[\hat{\boldsymbol{Q}} \cdot(\boldsymbol{h}+\boldsymbol{k})]\}\left\{3 \tilde{g}^{\prime 2}-\left[2\left(\hat{\boldsymbol{k}} \cdot \widehat{\boldsymbol{k}^{\prime}}\right)^{2}-1\right] \widetilde{h}^{\prime 2}+2 \widetilde{g}^{\prime} \widetilde{h}^{\prime}\right\}\right. \\
& +\left(-3 \mu_{s}^{2}+\mu_{v}{ }^{2}\right)\left\{Q^{2} \widetilde{g}^{\prime 2}+\left[\left(\boldsymbol{Q} \cdot \boldsymbol{k}^{\prime}\right)^{2}-2\left(\boldsymbol{k} \cdot \boldsymbol{k}^{\prime}\right)(\boldsymbol{Q} \cdot \boldsymbol{k})\left(\boldsymbol{Q} \cdot \boldsymbol{k}^{\prime}\right)\right] \widetilde{h}^{\prime 2}+\left(\boldsymbol{Q} \cdot \widehat{\boldsymbol{k}^{\prime}}\right)^{2} \tilde{g}^{\prime}(k) \widetilde{h}^{\prime}\left(k^{\prime}\right)\right. \\
& \left.\left.+\left[Q^{2}-2(\boldsymbol{Q} \cdot \hat{\boldsymbol{k}})^{2}\right] \tilde{g}^{\prime}\left(k^{\prime}\right) \tilde{h}^{\prime}(k)\right\}-\left(-3 \mu_{s}+\mu_{v}\right) \tilde{h}^{\prime 2} \frac{2}{Q^{2}}\left(\hat{\boldsymbol{k}} \cdot \widehat{\boldsymbol{k}^{\prime}}\right)\left[(\hat{\boldsymbol{k}} \cdot \boldsymbol{Q}) \widehat{\boldsymbol{k}^{\prime}} \cdot(2 \boldsymbol{h}+\boldsymbol{k})-\left(\widehat{\boldsymbol{k}^{\prime}} \cdot \boldsymbol{Q}\right) \hat{\boldsymbol{k}} \cdot(2 \boldsymbol{h}+\boldsymbol{k})\right]\right\} .
\end{aligned}
$$

First-order exchange contribution to the RPA-type correlation [see Eq. (22) and graph RPA1E of Fig. 1]:

$$
\begin{aligned}
{\left[S_{\mathrm{RPA} 1 \mathrm{E}}(\boldsymbol{Q}, \nu)\right]_{L, T}=} & -\frac{A}{(2 \pi)^{3}}\left(\frac{f_{\pi}^{2}}{4 \pi \hbar c}\right) \frac{3\left(m c^{2}\right)^{2}}{2\left(\hbar c \mu_{\pi}\right)^{2} \hbar c k_{F}} \int d^{3} h \int d^{3} k \mathcal{O} \mathcal{V}_{(L, T), \mathrm{RPA} 1 \mathrm{E}} \theta(1-h) \theta(|\boldsymbol{h}+\boldsymbol{Q}|-1) \\
& \times \theta(1-|\boldsymbol{h}+\boldsymbol{k}|) \theta(|\boldsymbol{h}+\boldsymbol{k}+\boldsymbol{Q}|-1)\left(-\frac{1}{\pi} \operatorname{Im}\right)\left\{\left(\frac{1}{2 \nu-\left(Q^{2}+2 \boldsymbol{h} \cdot \boldsymbol{Q}\right)+i \eta}-\frac{1}{2 \nu+\left(Q^{2}+2 \boldsymbol{h} \cdot \boldsymbol{Q}\right)}\right)\right. \\
& \left.\times\left(\frac{1}{2 \nu-\left[Q^{2}+2(\boldsymbol{h}+\boldsymbol{k}) \cdot \boldsymbol{Q}\right]+i \eta}-\frac{1}{2 \nu+\left[Q^{2}+2(\boldsymbol{h}+\boldsymbol{k}) \cdot \boldsymbol{Q}\right]}\right)\right\},
\end{aligned}
$$

where

$$
\mathcal{O} \mathcal{V}_{(L), \operatorname{RPA} 1 \mathrm{E}}=\Gamma_{\pi}^{2}(k)\left(3 \tilde{g}^{\prime}+\tilde{h}^{\prime}\right)
$$

and

$$
\mathcal{O} \mathcal{V}_{(T), \mathrm{RPA} 1 \mathrm{E}}=\left(\frac{\hbar c k_{F}}{2 m c^{2}}\right)^{2} \Gamma_{\pi}^{2}(k)\left(\left[3 \tilde{g}^{\prime}+\tilde{h}^{\prime}\right] 4\left\{[\boldsymbol{h} \cdot(\boldsymbol{h}+\boldsymbol{k})-(\boldsymbol{Q} \cdot \boldsymbol{h}) \boldsymbol{Q} \cdot(\boldsymbol{h}+\boldsymbol{k})] / Q^{2}\right\}+\left(-3 \mu_{s}{ }^{2}+\mu_{v}{ }^{2}\right) Q^{2}\left[\tilde{g}^{\prime}+\tilde{h}^{\prime}(\hat{\boldsymbol{k}} \cdot \hat{\boldsymbol{Q}})^{2}\right]\right) .
$$

Graph RPA2E of Fig. 1 [see Eq. (23)]: 


$$
\begin{aligned}
{\left[S_{\mathrm{RPA} 2 \mathrm{E}}(\boldsymbol{Q}, \nu)\right]_{T}=} & -\frac{A}{(2 \pi)^{5}}\left(\frac{f_{\pi}^{2}}{4 \pi \hbar c}\right)^{2} \frac{3\left(m c^{2}\right)^{3}}{\left(\hbar c \mu_{\pi}\right)^{4}}\left(-\frac{1}{\pi} \operatorname{Im}\right)\left\{\mathcal{L}(\boldsymbol{Q}, \nu) \int d^{3} h \int d^{3} k \mathcal{O V}_{(L, T), \mathrm{RPA} 2 \mathrm{E}} \theta(1-h) \theta(|\boldsymbol{h}+\boldsymbol{Q}|-1)\right. \\
& \times \theta(1-|\boldsymbol{h}+\boldsymbol{k}|) \theta(|\boldsymbol{h}+\boldsymbol{k}+\boldsymbol{Q}|-1)\left(\frac{1}{2 \nu-\left(Q^{2}+2 \boldsymbol{h} \cdot \boldsymbol{Q}\right)+i \eta}-\frac{1}{2 \nu+\left(Q^{2}+2 \boldsymbol{h} \cdot \boldsymbol{Q}\right)}\right) \\
& \left.\times\left(\frac{1}{2 \nu-\left[Q^{2}+2(\boldsymbol{h}+\boldsymbol{k}) \cdot \boldsymbol{Q}\right]+i \eta}-\frac{1}{2 \nu+\left[Q^{2}+2(\boldsymbol{h}+\boldsymbol{k}) \cdot \boldsymbol{Q}\right]}\right)\right\}
\end{aligned}
$$

with

$$
\mathcal{L}(\boldsymbol{Q}, \nu)=\int d^{3} p \theta(|\boldsymbol{p}+\boldsymbol{Q} / 2|-1) \theta(1-|\boldsymbol{p}-\boldsymbol{Q} / 2|)\left(\frac{1}{2 \nu-Q^{2}-2 \boldsymbol{Q} \cdot \boldsymbol{p}+i \eta}-\frac{1}{2 \nu+Q^{2}+2 \boldsymbol{Q} \cdot \boldsymbol{p}}\right)
$$

and

$$
\mathcal{O} \mathcal{V}_{(T), \operatorname{RPA} 2 \mathrm{E}}=\Gamma_{\pi}^{2}(Q) \Gamma_{\pi}^{2}(k) Q^{2} 4 \tilde{g}^{\prime} \mu_{v}^{2}\left[\tilde{g}^{\prime}+\tilde{h}^{\prime}(\hat{\boldsymbol{k}} \cdot \hat{\boldsymbol{Q}})^{2}\right]
$$

(Note that for the present interaction the longitudinal contribution is zero.)

Graph RPA2E' of Fig. 1 [see Eq. (23)]:

$$
\begin{aligned}
{\left[S_{\mathrm{RPA} 2 \mathrm{E}^{\prime}}(\boldsymbol{Q}, \nu)\right]_{L, T}=} & \frac{A}{(2 \pi)^{5}}\left(\frac{f_{\pi}^{2}}{4 \pi \hbar c}\right)^{2} \frac{6 m c^{2} k_{F}^{2}}{\left(\hbar c \mu_{\pi}^{2}\right)^{2}} \int d^{3} h \int d^{3} k \int d^{3} k^{\prime} \mathcal{O} \mathcal{V}_{(L, T), \mathrm{RPA}^{\prime}} \theta(1-h) \theta(|\boldsymbol{h}+\boldsymbol{Q}|-1) \theta(1-|\boldsymbol{h}+\boldsymbol{k}|) \\
& \times \theta(|\boldsymbol{h}+\boldsymbol{k}+\boldsymbol{Q}|-1) \theta\left(1-\left|\boldsymbol{h}+\boldsymbol{k}+\boldsymbol{k}^{\prime}\right|\right) \theta\left(\left|\boldsymbol{h}+\boldsymbol{k}+\boldsymbol{k}^{\prime}+\boldsymbol{Q}\right|-1\right)\left(-\frac{1}{\pi} \operatorname{Im}\right)\left\{\left(\frac{1}{2 \nu-\left(Q^{2}+2 \boldsymbol{h} \cdot \boldsymbol{Q}\right)+i \eta}\right.\right. \\
& \left.-\frac{1}{2 \nu+\left(Q^{2}+2 \boldsymbol{h} \cdot \boldsymbol{Q}\right)}\right)\left(\frac{1}{2 \nu-\left[Q^{2}+2(\boldsymbol{h}+\boldsymbol{k}) \cdot \boldsymbol{Q}\right]+i \eta}-\frac{1}{2 \nu+\left(Q^{2}+2(\boldsymbol{h}+\boldsymbol{k}) \cdot \boldsymbol{Q}\right)}\right) \\
& \left.\times\left(\frac{1}{2 \nu-\left[Q^{2}+2\left(\boldsymbol{h}+\boldsymbol{k}+\boldsymbol{k}^{\prime}\right) \cdot \boldsymbol{Q}\right]+i \eta}-\frac{1}{2 \nu+\left[Q^{2}+2\left(\boldsymbol{h}+\boldsymbol{k}+\boldsymbol{k}^{\prime}\right) \cdot \boldsymbol{Q}\right]}\right)\right\},
\end{aligned}
$$

where

$$
\mathcal{O} \mathcal{V}_{(L), \mathrm{RPA} 2 \mathrm{E}^{\prime}}=\Gamma_{\pi}^{2}(k) \Gamma_{\pi}^{2}\left(k^{\prime}\right) 10\left[9\left(\tilde{g}^{\prime}\right)^{2}+\tilde{h}^{\prime 2}+6 \tilde{g}^{\prime} \tilde{h}^{\prime}\right]
$$

and

$$
\begin{aligned}
\mathcal{O} \mathcal{V}_{(T), \mathrm{RPA} 2 \mathrm{E}^{\prime}}= & \left(\frac{\hbar c k_{F}}{2 m c^{2}}\right)^{2} \Gamma_{\pi}^{2}(k) \Gamma_{\pi}^{2}\left(k^{\prime}\right)\left\{40\left[9\left(\tilde{g}^{\prime}\right)^{2}+\tilde{h}^{\prime 2}+6{\widetilde{g^{\prime}}}^{\prime}\right]\left\{\left[\boldsymbol{h} \cdot\left(\boldsymbol{h}+\boldsymbol{k}+\boldsymbol{k}^{\prime}\right)-(\boldsymbol{Q} \cdot \boldsymbol{h}) \boldsymbol{Q} \cdot\left(\boldsymbol{h}+\boldsymbol{k}+\boldsymbol{k}^{\prime}\right)\right] / Q^{2}\right\}\right. \\
& +\frac{9 \mu_{s}^{2}+\mu_{v}^{2}}{2}\left(Q^{2}\left\{\left(\widetilde{g}_{2}^{\prime}\right)^{2}+\tilde{h}^{\prime 2}\left[2\left(\hat{\boldsymbol{k}} \cdot \widehat{\boldsymbol{k}^{\prime}}\right)^{2}-1\right]\right\}\right)+\widetilde{h}^{\prime 2}\left[(\boldsymbol{Q} \cdot \hat{\boldsymbol{k}})^{2}+\left(\boldsymbol{Q} \cdot \widehat{\boldsymbol{k}^{\prime}}\right)^{2}-2(\boldsymbol{Q} \cdot \hat{\boldsymbol{k}})\left(\boldsymbol{Q} \cdot \widehat{\boldsymbol{k}^{\prime}}\right)\left(\hat{\boldsymbol{k}} \cdot \widehat{\boldsymbol{k}^{\prime}}\right)\right] \\
& \left.+\widetilde{g}_{2}^{\prime} \widetilde{h}^{\prime}\left[(\boldsymbol{Q} \cdot \hat{\boldsymbol{k}})^{2}+\left(\boldsymbol{Q} \cdot \widehat{\boldsymbol{k}^{\prime}}\right)^{2}\right]\right\} .
\end{aligned}
$$

Going now to the $S_{Q P}$ and $S_{Q Q}$ channels [see Eqs. (26) and (27)] we have for graph $S Q P E$ of Fig. 2

$$
\begin{aligned}
{\left[S_{S Q P E}(\boldsymbol{Q}, \nu)\right]_{L, T}=} & \frac{A}{(2 \pi)^{5}}\left(\frac{f_{\pi}^{2}}{4 \pi \hbar c}\right)^{2} \frac{3\left(m c^{2}\right)^{3}}{4\left(\hbar c \mu_{\pi}\right)^{4}} \int d^{3} h \int d^{3} k \int d^{3} k^{\prime} \mathcal{O} \mathcal{V}_{(L, T), S Q P E} \theta(1-|\boldsymbol{h}|) \theta(|\boldsymbol{h}-\boldsymbol{k}|-1) \\
& \times \theta(|\boldsymbol{h}-\boldsymbol{k}+\boldsymbol{Q}|-1) \theta\left(\left|\boldsymbol{h}-\boldsymbol{k}^{\prime}+\boldsymbol{Q}\right|-1\right) \theta(|\boldsymbol{h}+\boldsymbol{Q}|-1) \theta\left(1-\left|\boldsymbol{h}-\boldsymbol{k}-\boldsymbol{k}^{\prime}+\boldsymbol{Q}\right|\right) \frac{1}{\boldsymbol{k} \cdot\left(\boldsymbol{k}^{\prime}-\boldsymbol{Q}\right)}\left(\frac{-1}{\pi} \operatorname{Im}\right) \\
& \times\left[\frac{1}{\nu-\left(Q^{2} / 2+\boldsymbol{Q} \cdot \boldsymbol{h}-\boldsymbol{k} \cdot \boldsymbol{k}^{\prime}\right)+i \eta} \frac{1}{\nu-\left(Q^{2} / 2+\boldsymbol{Q} \cdot \boldsymbol{h}\right)+i \eta}\right]
\end{aligned}
$$

Graph $S Q P E^{\prime}$ of Fig. 2: 


$$
\begin{aligned}
{\left[S_{S Q P E^{\prime}}(\boldsymbol{Q}, \nu)\right]_{L, T}=} & \frac{A}{(2 \pi)^{5}}\left(\frac{f_{\pi}^{2}}{4 \pi \hbar c}\right)^{2} \frac{3\left(m c^{2}\right)^{3}}{4\left(\hbar c \mu_{\pi}\right)^{4}} \int d^{3} h \int d^{3} k \int d^{3} k^{\prime} \mathcal{O} V_{(L, T), S Q P E^{\prime}} \theta(1-|\boldsymbol{h}|) \theta(|\boldsymbol{h}-\boldsymbol{k}|-1) \\
& \times \theta\left(\left|\boldsymbol{h}-\boldsymbol{k}^{\prime}+\boldsymbol{Q}\right|-1\right) \theta(|\boldsymbol{h}+\boldsymbol{Q}|-1) \theta\left(\left|\boldsymbol{h}-\boldsymbol{k}^{\prime}\right|-1\right) \theta\left(1-\left|\boldsymbol{h}-\boldsymbol{k}-\boldsymbol{k}^{\prime}\right|\right) \frac{1}{\boldsymbol{k} \cdot \boldsymbol{k}^{\prime}}\left(\frac{-1}{\pi} \operatorname{Im}\right) \\
& \times\left[\frac{1}{\nu-\left[Q^{2} / 2+\boldsymbol{Q} \cdot \boldsymbol{h}-\boldsymbol{k}^{\prime} \cdot(\boldsymbol{k}+\boldsymbol{Q})\right]+i \eta} \frac{1}{\nu-\left(Q^{2} / 2+\boldsymbol{Q} \cdot \boldsymbol{h}\right)+i \eta}\right],
\end{aligned}
$$

where

$$
\begin{aligned}
& \mathcal{O} \mathcal{V}_{L, S Q P E}=\mathcal{O} \mathcal{V}_{L, \mathrm{SE} 2 \mathrm{E}} \\
& \mathcal{O} \mathcal{V}_{T, S Q P E}=\mathcal{O} \mathcal{V}_{T, \mathrm{SE} 2 \mathrm{E}} \\
& \mathcal{O} \mathcal{V}_{T, S Q P E^{\prime}}=\left(\frac{\hbar c k_{F}}{2 m c^{2}}\right)^{2} \Gamma_{\pi}^{2}(k) \Gamma_{\pi}^{2}\left(k^{\prime}\right)\left(4\left\{\boldsymbol{h} \cdot\left(\boldsymbol{h}+\boldsymbol{k}^{\prime}\right)-(\hat{\boldsymbol{Q}} \cdot \boldsymbol{h})\left[\hat{\boldsymbol{Q}} \cdot\left(\boldsymbol{h}+\boldsymbol{k}^{\prime}\right)\right]\right\}\left\{3 \tilde{g}^{\prime 2}-\left[2\left(\hat{\boldsymbol{k}} \cdot \widehat{\boldsymbol{k}^{\prime}}\right)^{2}-1\right] \tilde{h}^{\prime 2}+2 \widetilde{g}^{\prime} \tilde{h}^{\prime}\right\}+\left(-3 \mu_{s}^{2}\right.\right. \\
& \left.+\mu_{v}{ }^{2}\right)\left\{Q^{2} \tilde{g}^{\prime 2}+\left[\left(\boldsymbol{Q} \cdot \boldsymbol{k}^{\prime}\right)^{2}-2\left(\boldsymbol{k} \cdot \boldsymbol{k}^{\prime}\right)(\boldsymbol{Q} \cdot \boldsymbol{k})\left(\boldsymbol{Q} \cdot \boldsymbol{k}^{\prime}\right)\right] \tilde{h}^{\prime 2}+\left(\boldsymbol{Q} \cdot \widehat{\boldsymbol{k}^{\prime}}\right)^{2} \tilde{g}^{\prime}(k) \tilde{h}^{\prime}\left(k^{\prime}\right)+\left[Q^{2}-2(\boldsymbol{Q} \cdot \hat{\boldsymbol{k}})^{2}\right] \tilde{g}^{\prime}\left(k^{\prime}\right) \tilde{h}^{\prime}(k)\right\} \\
& \left.-\left(-3 \mu_{s}+\mu_{v}\right) \widetilde{h}^{\prime 2} \frac{2}{Q^{2}}\left(\hat{\boldsymbol{k}} \cdot \widehat{\boldsymbol{k}^{\prime}}\right)\left[(\hat{\boldsymbol{k}} \cdot \boldsymbol{Q}) \widehat{\boldsymbol{k}^{\prime}} \cdot\left(2 \boldsymbol{h}+\boldsymbol{k}^{\prime}\right)-\left(\widehat{\boldsymbol{k}^{\prime}} \cdot \boldsymbol{Q}\right) \hat{\boldsymbol{k}} \cdot\left(2 \boldsymbol{k}+\boldsymbol{k}^{\prime}\right)\right]\right) . \\
& {\left[S_{Q P D^{\prime}}(\boldsymbol{Q}, \nu)\right]_{L, T}=-\frac{A}{(2 \pi)^{5}}\left(\frac{f_{\pi}^{2}}{4 \pi \hbar c}\right)^{2} \frac{3\left(m c^{2}\right)^{3}}{2\left(\hbar c \mu_{\pi}\right)^{4}} \int d^{3} h \int d^{3} h^{\prime} \int d^{3} k \mathcal{O} \mathcal{V}_{(L, T), S Q P D^{\prime}} \theta(1-|\boldsymbol{h}|) \theta(|\boldsymbol{h}+\boldsymbol{Q}|-1)} \\
& \times \theta(|\boldsymbol{h}-\boldsymbol{k}|-1) \theta\left(\left|\boldsymbol{h}^{\prime}+\boldsymbol{k}+\boldsymbol{Q}\right|-1\right) \theta\left(\left|\boldsymbol{h}^{\prime}+\boldsymbol{k}\right|-1\right) \theta\left(1-\left|\boldsymbol{h}^{\prime}\right|\right) \frac{1}{k^{2}-\boldsymbol{k} \cdot \boldsymbol{h}+\boldsymbol{k} \cdot \boldsymbol{h}^{\prime}}\left(\frac{-1}{\pi} \operatorname{Im}\right) \\
& \times \frac{1}{\nu-\left(Q^{2} / 2+\boldsymbol{Q} \cdot \boldsymbol{h}\right)+i \eta} \frac{1}{\nu-\left[k^{2}+Q^{2} / 2+\boldsymbol{k} \cdot\left(\boldsymbol{h}^{\prime}-\boldsymbol{h}\right)+\boldsymbol{Q} \cdot\left(\boldsymbol{k}+\boldsymbol{h}^{\prime}\right)\right]+i \eta} .
\end{aligned}
$$

Graph $S Q Q 3 D^{\prime}$ of Fig. 3:

$$
\begin{aligned}
{\left[S_{Q Q 3 D^{\prime}}(\boldsymbol{Q}, \nu)\right]_{L, T}=} & \frac{A}{(2 \pi)^{5}}\left(\frac{f_{\pi}^{2}}{4 \pi \hbar c}\right)^{2} \frac{3\left(m c^{2}\right)^{3}}{2\left(\hbar c \mu_{\pi}\right)^{4}} \int d^{3} h \int d^{3} h^{\prime} \int d^{3} k \mathcal{O} \mathcal{V}_{(L, T), S Q Q 3 D^{\prime}} \theta(1-|\boldsymbol{h}|) \theta(|\boldsymbol{h}-\boldsymbol{k}|-1) \\
& \times \theta\left(1-\left|\boldsymbol{h}^{\prime}+\boldsymbol{Q}\right|\right) \theta(|\boldsymbol{h}-\boldsymbol{k}+\boldsymbol{Q}|-1) \theta\left(\left|\boldsymbol{h}^{\prime}+\boldsymbol{k}\right|-1\right) \\
& \times \theta\left(1-\left|\boldsymbol{h}^{\prime}\right|\right) \frac{1}{k^{2}-\boldsymbol{k} \cdot \boldsymbol{h}+\boldsymbol{k} \cdot \boldsymbol{h}^{\prime}} \frac{1}{k^{2}+\boldsymbol{k} \cdot\left(\boldsymbol{h}^{\prime}-\boldsymbol{h}\right)+\boldsymbol{Q} \cdot\left(\boldsymbol{h}-\boldsymbol{h}^{\prime}-\boldsymbol{k}\right)} \\
& \times\left(\frac{-1}{\pi} \operatorname{Im}\right) \frac{1}{\nu-\left[k^{2}+Q^{2} / 2+\boldsymbol{k} \cdot\left(\boldsymbol{h}^{\prime}-\boldsymbol{h}\right)+\boldsymbol{Q} \cdot(\boldsymbol{h}-\boldsymbol{k})\right]+i \eta}
\end{aligned}
$$

In Eqs. (B19),(B20), we have used

$$
\boldsymbol{k}^{\prime}=\boldsymbol{k}-\boldsymbol{Q}
$$

and

$$
\mathcal{O} \mathcal{V}_{L, S Q P D^{\prime}}=\mathcal{O} \mathcal{V}_{L, S Q Q 3 D^{\prime}}=\Gamma_{\pi}^{2}(k) \Gamma_{\pi}^{2}\left(k^{\prime}\right) 5\left\{3 \tilde{g}^{\prime 2}+\left(\hat{\boldsymbol{k}} \cdot \widehat{\boldsymbol{k}^{\prime}}\right)^{2} \widetilde{h}^{\prime 2}+2 \tilde{g}^{\prime} \widetilde{h}^{\prime}\right\}
$$




$$
\begin{aligned}
\mathcal{O} \mathcal{V}_{T, S Q P D^{\prime}}= & \left(\frac{\hbar c k_{F}}{2 m c^{2}}\right)^{2} \Gamma_{\pi}^{2}(k) \Gamma_{\pi}^{2}\left(k^{\prime}\right)\left\{20\left\{\boldsymbol{h} \cdot\left(\boldsymbol{h}^{\prime}+\boldsymbol{k}\right)-(\hat{\boldsymbol{Q}} \cdot \boldsymbol{h})\left[\hat{\boldsymbol{Q}} \cdot\left(\boldsymbol{h}^{\prime}+\boldsymbol{k}\right)\right]\right\}\left[3 \tilde{g}^{\prime 2}+\left(\hat{\boldsymbol{k}} \cdot \widehat{\boldsymbol{k}^{\prime}}\right)^{2} \widetilde{h}^{\prime 2}+2 \tilde{g}^{\prime} \widetilde{h}^{\prime}\right]+\left(3 \mu_{s}^{2}+2 \mu_{v}^{2}\right)\right. \\
& \times\left\{4 Q^{2} \widetilde{g}^{\prime 2}+\left[\boldsymbol{Q} \times\left(\boldsymbol{k} \times \boldsymbol{k}^{\prime}\right)\right]^{2} \widetilde{h}^{\prime 2}+\left[Q^{2}+\left(\boldsymbol{Q} \cdot \widehat{\boldsymbol{k}^{\prime}}\right)^{2}\right] \widetilde{g}^{\prime}(k) \widetilde{h}^{\prime}\left(k^{\prime}\right)+\left[Q^{2}+(\boldsymbol{Q} \cdot \hat{\boldsymbol{k}})^{2}\right] \tilde{g}^{\prime}\left(k^{\prime}\right) \widetilde{h}^{\prime}(k)\right\} \\
& \left.-\left(3 \mu_{s}+2 \mu_{v}\right) \widetilde{h}^{\prime 2} \frac{2}{Q^{2}}\left(\hat{\boldsymbol{k}} \cdot \widehat{\boldsymbol{k}^{\prime}}\right)\left\{(\hat{\boldsymbol{k}} \cdot \boldsymbol{Q})\left[\widehat{\boldsymbol{k}^{\prime}} \cdot\left(\boldsymbol{h}+\boldsymbol{h}^{\prime}+\boldsymbol{k}\right)\right]-\left(\widehat{\boldsymbol{k}^{\prime}} \cdot \boldsymbol{Q}\right)\left[\hat{\boldsymbol{k}} \cdot\left(\boldsymbol{h}+\boldsymbol{h}^{\prime}+\boldsymbol{k}^{\prime}\right)\right]\right\}\right\}
\end{aligned}
$$

and

$$
\begin{aligned}
\mathcal{O} \mathcal{V}_{T, S Q Q 3 D^{\prime}}= & \left(\frac{\hbar c k_{F}}{2 m c^{2}}\right)^{2} \Gamma_{\pi}^{2}(k) \Gamma_{\pi}^{2}\left(k^{\prime}\right)\left\{20\left\{\boldsymbol{h}^{\prime} \cdot(\boldsymbol{h}-\boldsymbol{k})-\left(\hat{\boldsymbol{Q}} \cdot \boldsymbol{h}^{\prime}\right)[\hat{\boldsymbol{Q}} \cdot(\boldsymbol{h}-\boldsymbol{k})]\right\}\left[3 \tilde{g}^{\prime 2}+\left(\hat{\boldsymbol{k}} \cdot \widehat{\boldsymbol{k}^{\prime}}\right)^{2} \widetilde{h}^{\prime 2}+2 \tilde{g}^{\prime} \widetilde{h}^{\prime}\right]+\left(3 \mu_{s}^{2}+2 \mu_{v}^{2}\right)\right. \\
& \times\left\{4 Q^{2} \widetilde{g}^{\prime 2}+\left[\boldsymbol{Q} \times\left(\boldsymbol{k} \times \boldsymbol{k}^{\prime}\right)\right]^{2} \widetilde{h}^{\prime 2}+\left[Q^{2}+\left(\boldsymbol{Q} \cdot \widehat{\boldsymbol{k}^{\prime}}\right)^{2}\right] \widetilde{g}^{\prime}(k) \widetilde{h}^{\prime}\left(k^{\prime}\right)+\left[Q^{2}+(\boldsymbol{Q} \cdot \hat{\boldsymbol{k}})^{2}\right] \tilde{g}^{\prime}\left(\boldsymbol{k}^{\prime}\right) \widetilde{h}^{\prime}(k)\right\} \\
& \left.-\left(3 \mu_{s}+2 \mu_{v}\right) \tilde{h}^{\prime 2} \frac{2}{Q^{2}}\left(\hat{\boldsymbol{k}} \cdot \widehat{\boldsymbol{k}^{\prime}}\right)\left\{(\hat{\boldsymbol{k}} \cdot \boldsymbol{Q})\left[\widehat{\boldsymbol{k}^{\prime}} \cdot\left(\boldsymbol{h}+\boldsymbol{h}^{\prime}-\boldsymbol{k}\right)\right]-\left(\widehat{\boldsymbol{k}^{\prime}} \cdot \boldsymbol{Q}\right)\left[\hat{\boldsymbol{k}} \cdot\left(\boldsymbol{h}+\boldsymbol{h}^{\prime}-\boldsymbol{k}^{\prime}\right)\right]\right\}\right\}
\end{aligned}
$$

Graph $S Q Q 1 E$ of Fig. 3:

$$
\begin{aligned}
{\left[S_{S Q Q 1 E}(\boldsymbol{Q}, \nu)\right]_{L, T}=} & -\frac{A}{(2 \pi)^{5}}\left(\frac{f_{\pi}^{2}}{4 \pi \hbar c}\right)^{2} \frac{9\left(m c^{2}\right)^{3}}{4\left(\hbar c \mu_{\pi}\right)^{4}} \int d^{3} p \int d^{3} k \int d^{3} k^{\prime} \mathcal{O} \mathcal{V}_{(L, T), S Q Q 1 E} \theta(|\boldsymbol{p}|-1) \theta(1-|\boldsymbol{p}+\boldsymbol{k}|) \\
& \times \theta(|\boldsymbol{p}+\boldsymbol{Q}|-1) \theta\left(\left|\boldsymbol{p}+\boldsymbol{k}+\boldsymbol{k}^{\prime}\right|-1\right) \frac{1}{\left(\boldsymbol{k} \cdot \boldsymbol{k}^{\prime}\right)^{2}}\left(\frac{-1}{\pi} \operatorname{Im}\right)\left[\frac{1}{\nu-\left(Q^{2} / 2+\boldsymbol{Q} \cdot \boldsymbol{p}+\boldsymbol{k}^{\prime} \cdot \boldsymbol{k}\right)+i \eta}\right] .
\end{aligned}
$$

Finally, graph $S Q Q 2 E$ of Fig. 3:

$$
\begin{aligned}
{\left[S_{S Q Q 2 E}(\boldsymbol{Q}, \nu)\right]_{L, T}=} & -\frac{A}{(2 \pi)^{5}}\left(\frac{f_{\pi}^{2}}{4 \pi \hbar c}\right)^{2} \frac{9\left(m c^{2}\right)^{3}}{4\left(\hbar c \mu_{\pi}\right)^{4}} \int d^{3} h \int d^{3} k \int d^{3} k^{\prime} \mathcal{O} \mathcal{V}_{(L, T), S Q Q 2 E} \theta(1-|\boldsymbol{h}|) \theta(|\boldsymbol{h}-\boldsymbol{k}|-1) \\
& \times \theta\left(\left|\boldsymbol{h}-\boldsymbol{k}^{\prime}\right|-1\right) \theta(1-|\boldsymbol{h}-\boldsymbol{Q}|) \theta\left(1-\left|\boldsymbol{h}-\boldsymbol{k}-\boldsymbol{k}^{\prime}\right|\right) \frac{1}{\left(\boldsymbol{k} \cdot \boldsymbol{k}^{\prime}\right)^{2}}\left(\frac{-1}{\pi} \operatorname{Im}\right)\left[\frac{1}{\nu-\left(Q^{2} / 2+\boldsymbol{Q} \cdot \boldsymbol{h}-\boldsymbol{k}^{\prime} \cdot \boldsymbol{k}\right)+i \eta}\right],
\end{aligned}
$$

where

$$
\begin{gathered}
\mathcal{O} \mathcal{V}_{L, S Q Q 1 E}=\mathcal{O V}_{L, S Q Q 2 E}=\mathcal{O \mathcal { V } _ { L , S E 2 E } ,} \\
\mathcal{O} \mathcal{V}_{T, S Q Q 1 E}=\left(\frac{\hbar c k_{F}}{2 m c^{2}}\right)^{2} \Gamma_{\pi}^{2}(k) \Gamma_{\pi}^{2}\left(k^{\prime}\right)\left\{\left[\boldsymbol{p}^{2}-(\hat{\boldsymbol{Q}} \cdot \boldsymbol{p})^{2}\right]+Q^{2}\left(\mu_{s}{ }^{2}+\mu_{v}{ }^{2}\right) / 4\right\}\left\{3 \tilde{g}^{\prime 2}-\left[2\left(\hat{\boldsymbol{k}} \cdot \widehat{\boldsymbol{k}^{\prime}}\right)^{2}-1\right] \widetilde{h}^{\prime 2}+2 \tilde{g}^{\prime} \widetilde{h}^{\prime}\right\},
\end{gathered}
$$

and

$$
\mathcal{O} \mathcal{V}_{T, S Q Q 2 E}=\left(\frac{\hbar c k_{F}}{2 m c^{2}}\right)^{2} \Gamma_{\pi}^{2}(k) \Gamma_{\pi}^{2}\left(k^{\prime}\right)\left\{\left[\boldsymbol{h}^{2}-(\hat{\boldsymbol{Q}} \cdot \boldsymbol{h})^{2}\right]+Q^{2}\left(\mu_{s}^{2}+\mu_{v}^{2}\right) / 4\right\}\left\{3 \tilde{g}^{\prime 2}-\left[2\left(\hat{\boldsymbol{k}} \cdot \widehat{\boldsymbol{k}^{\prime}}\right)^{2}-1\right] \widetilde{h}^{\prime 2}+2 \tilde{g}^{\prime} \widetilde{h}^{\prime}\right\}
$$

[1] S. Boffi, C. Giusti, F. Pacati, and M. Radici, in Electromagnetic Response of Atomic Nuclei (Clarendon, Oxford 1996).

[2] P. Barreau et al., Nucl. Phys. A402, 515 (1983).

[3] Z. E. Meziani et al., Phys. Rev. Lett. 52, 2130 (1984).

[4] Z. E. Meziani et al., Phys. Rev. Lett. 54, 1233 (1985).

[5] C. C. Blatchley, J. J. LeRose, O. E. Pruet, D. Zimmermann, C. F. Williamsom, and M. Deady, Phys. Rev. C 34, 1243 (1986).
[6] C. F. Williamson (private communication).

[7] J. Jourdan, Nucl. Phys. A603, 117 (1996).

[8] J. V. Noble, Phys. Rev. Lett. 46, 412 (1981); Phys. Rev. C 27, 423 (1983).

[9] G. Do Dang and Nguyen van Giai, Phys. Rev. C 30, 731 (1984).

[10] H. Kurasawa and T. Suzuki, Nucl. Phys. A454, 527 (1986). 
[11] E. N. Nikolov, M. Bergmann, Chr. V. Christov, K. Goeke, A. N. Antonov, and S. Krewald, Phys. Lett. B 281, 208 (1992).

[12] M. Cavinato, D. Drechsel, E. Fein, M. Marangoni, and A. M. Saruis, Nucl. Phys. A423, 376 (1984).

[13] S. Drożdż, G. Co', J. Wambach, and J. Speth, Phys. Lett. B 185, 287 (1987).

[14] G. Co', K. F. Quader, R. D. Smith, and J. Wambach, Nucl. Phys. A485, 61 (1988).

[15] S. Drożdż, S. Nishizaki, J. Speth, and J. Wambach, Phys. Rep. 197, 1 (1990).

[16] A. Fabrocini and S. Fantoni, Nucl. Phys. A503, 375 (1989); A. Fabrocini, Phys. Rev. C 55, 338 (1997).

[17] Y. Horikawa, F. Lenz, and Nimai C. Mukhopadhyay, Phys. Rev. C 22, 1680 (1980).

[18] C. R. Chinn, A. Picklesimer, and J. W. Van Orden, Phys. Rev. C 40, 790 (1989).

[19] A. Picklesimer, J. W. Van Orden, and S. J. Wallace, Phys. Rev. C 32, 1312 (1985); C. R. Chinn, A. Picklesimer, and J. W. Van Orden, ibid. 40, 1159 (1989).

[20] F. Capuzzi, C. Giusti, and F. D. Pacati, Nucl. Phys. A524, 681 (1991)

[21] F. Capuzzi, Nucl. Phys. A554, 362 (1993).

[22] P. M. Boucher, B. Castel, and Y. Okuhara, Ann. Phys. (N.Y.) 196, 150 (1989).

[23] W. M. Alberico, M. Ericson, and A. Molinari, Ann. Phys. (N.Y.) 154, 356 (1984).
[24] W. M. Alberico, A. De Pace, A. Drago, and A. Molinari, Riv. Nuovo Cimento 14, 1 (1991).

[25] K. Takayanagi, K. Shimizu, and A. Arima, Nucl. Phys. A477, 205 (1988).

[26] K. Takayanagi, Nucl. Phys. A522, 523 (1991).

[27] K. Takayanagi, Phys. Lett. B 230, 11 (1989); Nucl. Phys. A510, 162 (1990); A522, 494 (1991); A556, 14 (1993).

[28] E. Bauer, Phys. Rev. C 43, 2438 (1991).

[29] A. De Pace and M. Viviani, Phys. Rev. C 48, 2931 (1993).

[30] E. Bauer, Nucl. Phys. A589, 669 (1995).

[31] E. Bauer, A. Ramos, and A. Polls, Phys. Rev. C 54, 2959 (1996).

[32] A. Polls, A. Ramos, and W. H. Dickhoff, in Two Nucleon Emission Reactions, edited by O. Benhar and A. Fabrocini (ETS, Pisa, 1990), p. 374.

[33] C. C. Gearhart, W. H. Dickhoff, A. Polls, and A. Ramos, Int. J. Mod. Phys. E 5, 461 (1996).

[34] A. Gil, J. Nieves, and E. Oset, Nucl. Phys. A627, 543 (1997).

[35] J. E. Amaro and A. M. Lallena, Nucl. Phys. A537, 585 (1992); J. E. Amaro, G. Co', and A. M. Lallena, Ann. Phys. (N.Y.) 221, 306 (1993).

[36] Y. Jin, D. S. Onley, and L. E. Wright, Phys. Rev. C 45, 1333 (1992).

[37] T. Shigehara, K. Shimizu, and A. Arima, Nucl. Phys. A492, 388 (1989).

[38] M. Buballa, S. Drożdż, S. Krewald, and J. Speth, Ann. Phys. (N.Y.) 208, 346 (1991). 